\title{
Second- and higher-order perturbations of a spherical spacetime
}

\author{
David Brizuela, José M. Martín-García, and Guillermo A. Mena Marugán \\ Instituto de Estructura de la Materia, CSIC, Serrano 121-123, 28006 Madrid, Spain
}

(Received 5 May 2006; published 30 August 2006)

\begin{abstract}
The Gerlach and Sengupta (GS) formalism of coordinate-invariant, first-order, spherical and nonspherical perturbations around an arbitrary spherical spacetime is generalized to higher orders, focusing on second-order perturbation theory. The GS harmonics are generalized to an arbitrary number of indices on the unit sphere and a formula is given for their products. The formalism is optimized for its implementation in a computer-algebra system, something that becomes essential in practice given the size and complexity of the equations. All evolution equations for the second-order perturbations, as well as the conservation equations for the energy-momentum tensor at this perturbation order, are given in covariant form, in Regge-Wheeler gauge.
\end{abstract}

DOI: 10.1103/PhysRevD.74.044039

PACS numbers: 04.25.Nx, 04.30.Db, 95.30.Sf

\section{INTRODUCTION}

When a physical problem cannot be solved exactly, one usually appeals to approximate methods. Perturbation theory provides one such approximate approach, allowing a description in terms of small departures around an exact solution. In the context of general relativity, perturbation theory plays a prominent role in analyzing and understanding dynamical processes, nowadays being an efficient and natural complement to full numerical relativity simulations [1].

In particular, perturbation theory is used to study the stability properties of solutions of interest: black hole spacetimes [2], cosmological solutions [3], critical solutions [4], and many others. It also allows us to check the presence of gauge instabilities [5], constraint violations [6], and other types of instabilities in the various formulations of the Einstein equations implemented in numerical relativity, since numerical errors can be considered themselves as distortions of the solution that one is computing. Of utmost importance, perturbation theory can provide us with estimates of the amount of gravitational radiation and of the signal profiles emitted in astrophysical scenarios like an oscillating neutron star [7], the gravitational collapse of a star [8], an extreme mass-ratio binary [9], or a close-limit head-on collision of two black holes [10].

The complexity of the expressions involved in the perturbation of the equations of general relativity is very high, rapidly increasing when working at higher orders of perturbation. Most of the previous investigations have been carried out at first order, and using highly symmetric unperturbed solutions, which simplifies the problem. Simple backgrounds have been perturbed at the second-order level: for example, the Friedmann-Robertson-Walker spacetime [11], the Kerr spacetime [12], or the Schwarzschild spacetime $[13,14]$. The change in the oscillation modes of a stationary star owing to its rotation has been studied in Refs. $[15,16]$, regarding the rotation as a firstorder perturbation of a static star (and therefore the change as a second-order effect). The critical exponent of angular momentum scaling has been predicted for scalar field collapse using second-order perturbation arguments [17]. Special credit should be given to the seminal work of Cunningham, Price, and Moncrief in 1980 where the second-order nonspherical perturbations of a collapsing star of dust were studied including the problem of matching of the internal and external perturbations through the surface of the star [18]. Second-order perturbations of the general problem of matching through a surface have been analyzed in Ref. [19].

Different reasons justify the importance of going beyond first-order perturbation theory. First, one must confront the obvious desire to reach higher accuracy in the numerical simulations of perturbative approximations to selfgravitating systems. In addition, second-order perturbations can estimate quantitatively the range of applicability of the first-order results by providing error estimates. Besides, they should enable us to study and interpret the nonlinearity of general relativity in terms of the coupling among first-order modes [20], a study that might allow us to model secular interactions which are too slow to be followed by using full numerical simulations or which might be incorrectly interpreted as small numerical errors.

In this work, we construct a generic framework to analyze second- and higher-order spherical and nonspherical perturbations of an arbitrary spherical spacetime, without restricting ourselves to any particular matter model, and show how to deal with the general case while keeping a still-manageable size for the resulting expressions. This work can be considered as a continuation of the work of Gerlach and Sengupta (GS) at first order [21,22], a formalism which was revived in Ref. [23] and is currently considered optimal for perturbations of generic spherical spacetimes [24,25] (even though for specific matter models the formalism might admit further simplifications). For example, its application to a perfect fluid spacetime with a two-parameter equation of state was considered in 
Ref. [26], including the matching of the fluid perturbations to an exterior spacetime through a moving timelike surface [27].

The GS formalism is based on four basic ingredients: a $2+2$ decomposition of the spacetime separating the spherical $S^{2}$ symmetry orbits from a general $1+1$ Lorentzian manifold $M^{2}$; the use of a covariant description both on the Lorentzian manifold and on the 2-sphere; the decomposition of the perturbations in $S^{2}$ tensor harmonics; and the use of gauge-invariant perturbation variables. The formalism developed in the present paper makes use of the first three ingredients, leaving the construction of gauge invariants for a future work. The use of a covariant notation is particularly convenient: on the one hand, it allows us to formulate all equations without choosing coordinates on $M^{2}$, something that becomes very useful on dynamical backgrounds; on the other hand, it eliminates all trigonometric factors from the equations of motion, factors which do not contain any relevant information and typically obscure the geometrical interpretation of the results. The use of covariant notation, however, makes computations more complicated. To overcome this problem we will intensively use computer-algebra tools, specially designed and developed by us for abstract tensor computations. All equations in this paper have been programmed and checked with those tools, resulting in a computer framework which allows us to work efficiently with applications of the formalism of high-order perturbations.

For some matter contents it is possible to further simplify the perturbative formalism by constructing scalar combinations of the perturbations which encode the purely dynamical degrees of freedom and obey evolution equations free of constraints (the so-called master equations for the master scalars). Among other matter models, this is possible for vacuum [28,29] and for the Maxwell field [30], but such master equations have not been found, for instance, for the case of a scalar field. Those scalars can be given in a gauge-invariant form [31] and expressed in a covariant form within the GS framework [21,32]. In this article we will concentrate on the construction of a generic perturbative formalism that can be applied to any background spherical spacetime. The construction of gaugeinvariant master scalars at high perturbative orders will be the subject of a future work.

The rest of this article is organized as follows. Section II introduces the fundamental concepts and equations of perturbation theory, and provides closed formulas for the $n$ th-order perturbations of the geometric quantities of interest (formulas that are new in the literature to the best of our knowledge). The notation used for the spherical background manifold is explained in Sec. III. Section IV introduces the Regge-Wheeler-Zerilli harmonics $[28,33]$ and generalizes them to arbitrary rank tensors. Formulas for their products are given, based on the representation matrices of the rotation group. Basically, nothing in that section is new, but it has been conveniently recast into GS notation, which proves to be very useful for applications and computational efficiency. Nonspherical perturbations are discussed in Sec. V, giving for the first time complete sources for the evolution equations of secondorder perturbations of a general spherical background, as well as sources for the energy-momentum conservation equations. Section VI contains a summary of our results and further discussions. Finally, several appendixes are added. They explain different aspects of the definition of spherical functions, the symmetric trace-free part of tensors, and the pure-orbital harmonics. They also provide the GS equations for first-order perturbation theory and describe the procedure followed to implement our calculations in MATHEMATICA [34].

\section{PERTURBATION THEORY IN GENERAL RELATIVITY}

In general relativity, one has to solve ten coupled nonlinear partial differential equations for the metric. Using perturbation theory, the problem can be reformulated as an infinite hierarchy of linear differential equations for the modification of the metric with respect to a known solution. Furthermore, in this hierarchy of equations, the principal parts are always given by the same differential operator acting on a perturbative correction of increasing order. In this way, one translates the difficulty from nonlinearity to the infinite number of equations. The key assumption of the perturbative scheme is that one can truncate the problem at a finite order and still obtain an approximate solution to the original system.

In order to introduce this perturbative hierarchy, let us start by considering an $\epsilon$ family of 4-dimensional metrics $\tilde{g}_{\mu \nu}(\epsilon)$ on a certain manifold $\mathcal{M}$, where $\epsilon$ is a dimensionless parameter [35]. The matter field content of these spacetimes provides another $\epsilon$ family which will be denoted abstractly by $\tilde{\Phi}(\epsilon)$. For simplicity, we assume that these families are smooth in the parameter $\epsilon$ (or at least $C^{n}$ with a locally Lipschitz $n$th derivative if we are interested only in perturbation theory up to order $n>0$ ). In particular, the metric and matter fields can be expanded as [36]

$$
\begin{gathered}
\tilde{g}_{\mu \nu}(\epsilon)=g_{\mu \nu}+\sum_{n=1}^{\infty} \frac{\epsilon^{n}}{n !}\{n\} h_{\mu \nu}, \\
\tilde{\Phi}(\epsilon)=\Phi+\sum_{n=1}^{\infty} \frac{\epsilon^{n}}{n !}\{n\}
\end{gathered}
$$

The $\epsilon=0$ fields $g_{\mu \nu}$ and $\Phi$ will be referred to as the "background" metric and matter. To distinguish the background objects from their "perturbed" $\epsilon \neq 0$ counterparts, we will denote the latter with a tilde ( ).

The coefficients ${ }^{\{n\}} h_{\mu \nu}$ and ${ }^{\{n\}} \Phi$ are tensors on the manifold $\mathcal{M}$ [37], and in what follows their indices are lowered or raised with the background metric $g_{\mu \nu}$ and its 
inverse $g^{\mu \nu}$. They can be obtained by repeated differentiation of the perturbed objects with respect to $\epsilon$. Actually, it proves most convenient to introduce a formal "perturbation" operator $\Delta$ with the properties of a derivative, so that any object $\tilde{T}(\epsilon)$ can be expanded as

$$
\tilde{T}(\epsilon)=T+\sum_{n=1}^{\infty} \frac{\epsilon^{n}}{n !} \Delta^{n}[T] .
$$

For instance, $\Delta\left[g_{\mu \nu}\right]=\{1\} h_{\mu \nu}$ and $\Delta\left[{ }^{\{n\}} h_{\mu \nu}\right]=\{n+1\} h_{\mu \nu}$. In this notation, the brackets are intended to avoid confusion with index positioning because, e.g., for a given vector $v^{\mu}$,

$$
\begin{aligned}
\Delta\left[v_{\mu}\right] & =\Delta\left[g_{\mu \nu} v^{\nu}\right]=g_{\mu \nu} \Delta\left[v^{\nu}\right]+\{1\} h_{\mu \nu} v^{\nu} \\
& \neq g_{\mu \nu} \Delta\left[v^{\nu}\right],
\end{aligned}
$$

so that the notation $\Delta v_{\mu}$ might be misleading.

The expansion for the inverse metric can be obtained by iteration of the identity

$$
\tilde{g}^{\mu \nu} \equiv g^{\mu \nu}-g^{\mu \lambda}\left(\tilde{g}_{\lambda \sigma}-g_{\lambda \sigma}\right) \tilde{g}^{\sigma \nu}
$$

or, equivalently, by repeated perturbation of the relation

$$
\Delta\left[g^{\mu \nu}\right]=-g^{\mu \alpha} \Delta\left[g_{\alpha \beta}\right] g^{\beta \nu} .
$$

This leads to

$$
\begin{aligned}
\tilde{g}^{\mu \nu}= & g^{\mu \nu}-\epsilon^{\{1\}} h^{\mu \nu}-\frac{\epsilon^{2}}{2}\left({ }^{\{2\}} h^{\mu \nu}-2^{\{1\}} h^{\mu \alpha\{1\}} h_{\alpha}{ }^{\nu}\right) \\
& +O\left(\epsilon^{3}\right),
\end{aligned}
$$

and, more generally, to a perturbation of the form

$$
\begin{aligned}
\Delta^{n}\left[g^{\mu \nu}\right]= & \sum_{\left(k_{i}\right)}(-1)^{m} \\
& \times \frac{n !}{k_{1} ! \ldots k_{m} !}{ }^{\left\{k_{m}\right\}} h^{\mu \alpha\left\{k_{m-1}\right\}} h_{\alpha \beta} \ldots{ }^{\left\{k_{2}\right\}} h_{\tau \rho}{ }^{\left\{k_{1}\right\}} h^{\rho \nu},
\end{aligned}
$$

where the sum extends to the $2^{n-1}$ sorted partitions of $n$ in $m \leq n$ positive integers $k_{1}+\cdots+k_{m}=n$. For example, for $n=4$ there are eight partitions: $(4),(1,3),(3,1),(2,2)$, $(1,1,2),(1,2,1),(2,1,1)$, and $(1,1,1,1)$.

The Christoffel symbols are

$$
\begin{aligned}
\tilde{\Gamma}_{\mu \nu}^{\alpha}= & \Gamma_{\mu \nu}^{\alpha}+\epsilon^{\{1\}} h_{\mu \nu}^{\alpha}+\frac{\epsilon^{2}}{2}\left({ }^{\{2\}} h_{\mu \nu}^{\alpha}-2^{\{1\}} h^{\alpha \beta\{1\}} h_{\beta \mu \nu}\right) \\
& +O\left(\epsilon^{3}\right)
\end{aligned}
$$

where we have defined the three-index perturbation

$$
{ }^{\{n\}} h_{\alpha \mu \nu} \equiv \frac{1}{2}\left({ }^{\{n\}} h_{\alpha \mu ; \nu}+{ }^{\{n\}} h_{\alpha \nu ; \mu}-\{n\} h_{\mu \nu ; \alpha}\right),
$$

which is symmetric in its last two indices and satisfies ${ }^{\{0\}} h_{\alpha \mu \nu}=0$. The covariant derivative in (10) is that associated with the background metric. Higher-order terms of the expansion can be easily computed noting that

$$
\Delta\left[{ }^{\{n\}} h_{\alpha \mu \nu}\right]=\{n+1\} h_{\alpha \mu \nu}-{ }^{\{n\}} h_{\alpha}{ }^{\beta\{1\}} h_{\beta \mu \nu},
$$

which, for $n>1$, leads to

$$
\begin{aligned}
\Delta^{n}\left[\Gamma_{\mu \nu}^{\alpha}\right]= & \sum_{\left(k_{i}\right)}(-1)^{m+1} \frac{n !}{k_{1} ! \ldots k_{m} !} \\
& \left.\times k_{m}\right\} h^{\alpha \beta\left\{k_{m-1}\right\}} h_{\beta \gamma} \ldots{ }^{\left\{k_{2}\right\}} h^{\tau \rho\left\{k_{1}\right\}} h_{\rho \mu \nu} .
\end{aligned}
$$

Here, the sum extends again to all sorted partitions of $n$. The case $n=1$ is special, with $\Delta\left[\Gamma^{\alpha}{ }_{\mu \nu}\right]=\left\{{ }^{11} h^{\alpha}{ }_{\mu \nu}\right.$. Note that each term in the above expression contains one and only one tensor ${ }^{\{k\}} h_{\rho \mu \nu}$, but a variable number of metric perturbations.

The perturbations of the Riemann tensor are given by

$$
\begin{aligned}
\tilde{R}_{\mu \nu \sigma}{ }^{\lambda}= & R_{\mu \nu \sigma^{\lambda}}+2 \epsilon^{\{1\}} h_{\sigma[\mu ; \nu]}^{\lambda}+\epsilon^{2}\left({ }^{\{2\}} h_{\sigma[\mu ; \nu]}^{\lambda}\right. \\
& \left.-2^{\{1\}} h^{\lambda \alpha\{1\}} h_{\alpha \sigma[\mu ; \nu]}+2^{\{1\}} h_{\alpha[\mu}{ }^{\lambda\{1\}} h^{\alpha}{ }_{\nu] \sigma}\right) \\
& +O\left(\epsilon^{3}\right),
\end{aligned}
$$

with the general term

$$
\begin{aligned}
\Delta^{n}\left[R_{\mu \nu \alpha}{ }^{\beta}\right]= & \nabla_{\nu}\left(\Delta^{n}\left[\Gamma^{\beta}{ }_{\alpha \mu}\right]\right)-\sum_{k=1}^{n-1}\left(\begin{array}{l}
n \\
k
\end{array}\right) \Delta^{k}\left[\Gamma_{\lambda \mu}^{\beta}\right] \\
& \times \Delta^{n-k}\left[\Gamma_{\nu \alpha}^{\lambda}\right]-(\mu \leftrightarrow \nu) .
\end{aligned}
$$

This expression does not involve the metric directly. That is, it only contains the background connection $\nabla$, and the perturbations of its Christoffel symbols, without assuming that either the background or the perturbed connections derive from a metric. Hence, it can be applied to the Palatini equations, for example. If the background connection derives from a metric, Eqs. (9)-(12) ensure that the perturbed connection also derives from a metric. Then, the (implicit) triple sum in Eq. (14) can be rearranged as follows:

$$
\begin{aligned}
\Delta^{n}\left[R_{\mu \nu \alpha}{ }^{\beta}\right]= & \sum_{\left(k_{i}\right)}(-1)^{m} \frac{n !}{k_{1} ! \ldots k_{m} !}\left[{ }^{\left\{k_{m}\right\}} h^{\beta \lambda_{m}} \ldots{ }^{\left\{k_{2}\right\}} h^{\lambda_{3} \lambda_{2}\left\{k_{1}\right\}} h_{\lambda_{2} \alpha \nu ; \mu}\right. \\
& \left.+\sum_{s=2}^{m}\left\{k_{m}\right\} h^{\beta \lambda_{m}} \ldots{ }^{\left\{k_{s+1}\right\}} h^{\lambda_{s+2} \lambda_{s+1}\left\{k_{s}\right\}} h_{\lambda_{s} \lambda_{s+1} \mu}{ }^{\left\{k_{s-1}\right\}} h^{\lambda_{s} \lambda_{s-1}} \ldots{ }^{\left\{k_{2}\right\}} h^{\lambda_{3} \lambda_{2}\left\{k_{1}\right\}} h_{\lambda_{2} \nu \alpha}\right]-(\mu \leftrightarrow \nu) .
\end{aligned}
$$


This formula is surprisingly simple because all covariant derivatives of the metric perturbations are grouped in $h$-terms of the form (10). It is clear that the explicit sum in Eq. (14) contains only that kind of term; however, the derivatives of the perturbations of the Christoffel symbols give rise to isolated covariant derivatives of the metric perturbations. Nonetheless, they can be combined with the sum to obtain the displayed result. Note also that this expression is already optimally simplified: all terms in the sums are generically different.

From the above formulas, we can compute the general perturbation of the Ricci and Einstein tensors. For Ricci, the perturbation is obtained from Eq. (15) by contracting the $\beta$ and $\nu$ indices. The resulting expression is symmetric in $\alpha$ and $\mu$ owing to the identity

$$
\sum_{k=1}^{n}{ }^{\{k\}} T^{\alpha \beta\{k\}} h_{\alpha \beta \mu ; \nu}=\sum_{k=1}^{n}{ }^{\{k\}} T^{\alpha \beta\{k\}} h_{\alpha \beta \nu ; \mu},
$$

valid for any family of symmetric tensors ${ }^{\{k\}} T^{\alpha \beta}$ such that $\sum_{k=1}^{n}{ }^{\{k\}} T^{\alpha \beta\{k\}} h_{\beta}{ }^{\mu}$ is also symmetric in $\alpha$ and $\mu$.

It is important to emphasize that these combinatorial formulas for the perturbations of the curvature tensors at a general order are extremely useful for computational purposes. Essentially, the problem of perturbations is reduced to that of listing the sorted partitions of a given number, which can be done very fast in any computer-algebra system. This fact simply reflects the recursive differential origin of the perturbation process. The formulas in this section have been implemented in the free package XPERT, briefly described in Appendix E.

\section{SPHERICAL BACKGROUND}

Following GS [21,22], the manifold of a spherical spacetime $\left(M^{4}, g_{\mu \nu}\right)$ is described as a product of the form $M^{4}=$ $M^{2} \times S^{2}$ using a coordinate system $x^{\mu}=\left(x^{A}, x^{a}\right)$ adapted to the $S^{2}$ orbits of spherical symmetry. The two scalars $x^{A}=\left\{x^{0}, x^{1}\right\}$ provide coordinates for the $1+1$ Lorentzian manifold $M^{2}$ with boundary, whereas $x^{a}=\left\{x^{2} \equiv \theta, x^{3} \equiv\right.$ $\phi\}$ are the usual spherical coordinates on the sphere $S^{2}$. The 4-dimensional metric $g_{\mu \nu}$ and the energy-momentum tensor $t_{\mu \nu}$ can always be written

$$
\begin{aligned}
g_{\mu \nu}\left(x^{D}, x^{d}\right) d x^{\mu} d x^{\nu}= & g_{A B}\left(x^{D}\right) d x^{A} d x^{B} \\
& +r^{2}\left(x^{D}\right) \gamma_{a b}\left(x^{d}\right) d x^{a} d x^{b}, \\
t_{\mu \nu}\left(x^{D}, x^{d}\right) d x^{\mu} d x^{\nu}= & t_{A B}\left(x^{D}\right) d x^{A} d x^{B} \\
& +\frac{1}{2} r^{2}\left(x^{D}\right) Q\left(x^{D}\right) \gamma_{a b}\left(x^{d}\right) d x^{a} d x^{b},
\end{aligned}
$$

where $r$ is a scalar field on $M^{2}, g_{A B}$ is a Lorentzian metric tensor on $M^{2}$, and $\gamma_{a b}$ is the round (unit Gaussian curvature) metric on $S^{2}$. Using this decomposition it is straightforward to express all 4-dimensional curvature tensors in terms of the curvature tensors of $g$ and $\gamma$ and the derivatives of the scalar $r$, as shown in Ref. [21]. In doing so, one usually introduces the vector field

$$
v_{A} \equiv \frac{r_{, A}}{r}=(\log r)_{, A}
$$

to avoid working with logarithms of $r$. Covariant derivatives of a vector $f$ on the manifolds $M^{4}, M^{2}$, and $S^{2}$ will be denoted as $f_{\mu ; \nu}, f_{A \mid B}$, and $f_{a: b}$, respectively. For instance, the Schwarzschild spacetime has been described along these lines in Refs. [24,25,32].

The totally antisymmetric tensors are denoted with the symbol $\epsilon$ and obey the conventions $\epsilon_{A B c d}=\epsilon_{A B} \epsilon_{c d}$ with $\epsilon_{01}=1$ on $M^{2}$ and $\epsilon_{23}=1$ on $S^{2}$. Note that some authors interchange the uppercase/lowercase index conventions, and others use the opposite sign for $\epsilon_{01}$.

On the other hand, as remarked by Newman and Penrose [38], it is convenient to introduce a basis of complex vectors on $S^{2}$ :

$$
m^{a}=\frac{1}{\sqrt{2}}\left(e_{\theta}{ }^{a}+i e_{\phi}{ }^{a}\right) \quad \text { and } \quad \bar{m}^{a}=\frac{1}{\sqrt{2}}\left(e_{\theta}{ }^{a}-i e_{\phi}{ }^{a}\right),
$$

where $e_{\theta}{ }^{a}$ and $e_{\phi}{ }^{a}$ are the unit norm (with respect to the round metric) basis vectors. From these vectors we obtain

$$
\bar{m}^{a} m^{b}=\frac{1}{2}\left(\gamma^{a b}+i \epsilon^{a b}\right),
$$

a relation which can be inverted to get

$$
\begin{gathered}
\gamma^{a b}=m^{a} \bar{m}^{b}+\bar{m}^{a} m^{b}, \\
\epsilon^{a b}=i\left(m^{a} \bar{m}^{b}-\bar{m}^{a} m^{b}\right) .
\end{gathered}
$$

These vectors are null, $\gamma_{a b} m^{a} m^{b}=\gamma_{a b} \bar{m}^{a} \bar{m}^{b}=0$, and are normalized so that $\gamma_{a b} m^{a} \bar{m}^{b}=1$.

\section{TENSOR HARMONICS}

The theory of tensor harmonics on the sphere $S^{2}$ is a very well-known subject [39-41]. In this section we recast it into the GS notation, which is particularly useful for the type of algebraic computations that must be performed in high-order perturbation theory. After a brief review of the GS harmonics we show that, in order to handle second and higher orders of perturbation, it is convenient to work with harmonics that possess an increasingly high number of indices. A generalization of the GS harmonics to many indices is then defined and shown to be closely related to the Wigner rotation matrices (also known as spin-weighted harmonics in general relativity [42]). Finally, we give closed formulas for arbitrary products of these harmonics. Appendix A explains our choice of conventions.

\section{A. GS notation for harmonics}

An orthonormal basis of functions on the sphere $S^{2}$ is given by the spherical harmonics $Y_{l}^{m}(\theta, \phi)$, which are 
defined as the eigenfunctions of the differential operators

$$
\begin{gathered}
\gamma^{a b} \nabla_{a} \nabla_{b} Y_{l}^{m}=-l(l+1) Y_{l}^{m}, \\
i \partial_{\phi} Y_{l}^{m}=-m Y_{l}^{m},
\end{gathered}
$$

where $\gamma^{a b}$ is the inverse of the round metric on $S^{2}$, the angle $\phi$ is defined by choosing a fixed $z$ axis, and $l$ and $m$ are integers such that $l \geq|m|$. These harmonics are normalized so that

$$
\int d \Omega Y_{l^{\prime}}^{m^{\prime}} Y_{l}^{m^{*}}=\delta_{l^{\prime} l} \delta_{m^{\prime} m}
$$

with $d \Omega$ being the area element on $S^{2}(d \Omega=\sin \theta d \theta d \phi)$.

From them, the Regge-Wheeler (RW) [28] basis of vector fields on the sphere can be defined as follows. The basis is formed by the vector fields $Y_{l}^{m}: a$ and their orthogonal fields $S_{l a}^{m} \equiv \epsilon_{a b} \gamma^{b c} Y_{l: c}^{m}$. A basis for 2-tensor fields on the sphere can also be constructed in a similar way [33] and it is formed by three types of objects: pure-trace tensors can be decomposed using $\gamma_{a b} Y_{l}^{m}$; antisymmetric tensors can be expanded using $\epsilon_{a b} Y_{l}^{m}$; and finally symmetric traceless tensors can be expanded using

$$
\begin{gathered}
Z_{l a b}^{m} \equiv\left(Y_{l: a b}^{m}\right)^{\mathrm{TF}}=Y_{l: a b}^{m}+\frac{l(l+1)}{2} \gamma_{a b} Y_{l}^{m}, \\
X_{l_{a b}}^{m} \equiv S_{l_{(a: b)}^{m} .}
\end{gathered}
$$

Indices in round brackets are symmetrized, and the superscript TF denotes the trace-free part. Note that GS use $2 S_{l(a: b)}^{m}$ instead of $X_{l a b}^{m}$, different by a factor of 2 .

\section{B. Products of harmonics}

In the next section we will expand the metric perturbations ${ }^{\{n\}} h_{\mu \nu}$ in tensor harmonics. From expressions like (8), (12), and (15) it is clear that we need to compute products of several tensor harmonics when working beyond linear perturbation theory. Even though those expressions contain products of many harmonics, the problem can be dealt with recursively because the product of two tensor harmonics can be decomposed as a series of tensor harmonics of adequate rank. In principle, we might conclude that at perturbation order $n$ we need to work with tensor harmonics of rank $2 n$ or similar, but the situation turns out to be simpler in general relativity.

The formalism starts from perturbations of the metric, which contain tensor harmonics on $S^{2}$ of rank zero, one, or two, and computes the decomposition in harmonics of the perturbations of the Einstein tensor, which also contain harmonics of those ranks. On the other hand, only second (at most) derivatives of the metric perturbations will appear in the perturbations of any curvature tensor, at any order. Finally, as long as we are interested just in perturbations of curvature tensors, only those contractions in Eq. (15) are required. From these three observations we conclude that we only need harmonics with up to four indices (if one works in RW gauge, to be defined below, only three-index harmonics are required) and formulas for their 13 products,

$$
\begin{aligned}
& Y Y^{\prime} \text {, } \\
& Y Y^{\prime}: a, \quad Y_{: a} Y_{: b}^{\prime}, \\
& Y Y^{\prime}: a b, \quad Y_{: a} Y_{: b c}^{\prime}, \quad Y_{: a b} Y_{: c d}^{\prime}, \\
& Y Y^{\prime}: a b c, \quad Y_{: a} Y^{\prime}: b c d, \quad Y_{: a b} Y_{: b c d}^{\prime}, \quad Y_{a b c} Y_{: d e f}^{\prime}, \\
& Y Y^{\prime}: a b c d, \quad Y_{: a} Y^{\prime}: b c d e, \quad Y_{: a b} Y^{\prime}: c d e f,
\end{aligned}
$$

where the primes denote that $Y$ and $Y^{\prime}$ have different labels $l$ and $m$. Only seven of those are really independent because using the Leibnitz rule we have relations like

$$
Y_{: a b} Y^{\prime}{ }_{c d}=\left(Y_{: a} Y^{\prime}{ }_{c d}\right)_{: b}-Y_{: a} Y^{\prime}{ }_{c d b} .
$$

Therefore, computing the expansion formula for the canonical products $Y Y^{\prime}: a_{1} \ldots a_{n}$ with $n=0, \ldots, 6$ would be enough to solve a general problem of nonspherical perturbations in general relativity.

That method would be, however, rather complicated to program, because it requires expanding the products of multiple harmonics in a very particular order, and it is difficult to use in any mathematical proof involving products of harmonics. It is far more interesting and general to follow a different route: we first generalize the GS harmonics to an arbitrary number of indices and then find a general formula for the product of any two of them. This has two important advantages: first, it is more efficient and simple for our algebraic code because all cases are considered in a single formula. Second, the formalism is more general: it can be applied to arbitrary matter models, it is possible to perturb objects like derivatives of the Riemann tensor, or it can be used in other problems (for example, theories of gravity with more than two derivatives in their basic equations).

\section{Higher-order generalization of GS tensors}

Complete bases for second- and higher-order tensors can be easily constructed. There always exist two nontrivial symmetric trace-free (STF) tensors,

$$
\begin{aligned}
& Z_{l a_{1} \ldots a_{s}}^{m} \equiv\left(Y_{l: a_{1} \ldots a_{s}}^{m}\right)^{\mathrm{STF}}=-\epsilon_{\left(a_{1}{ }^{b} X_{l}{ }^{m} b a_{2} \ldots a_{s}\right)}, \\
& X_{l a_{1} \ldots a_{s}}^{m} \equiv\left(S_{l a_{1}: a_{2} \ldots a_{s}}^{m}\right)^{\mathrm{STF}}=\epsilon_{\left(a_{1}\right.}{ }^{b} Z_{\left.l b a_{2} \ldots a_{s}\right)}^{m},
\end{aligned}
$$

valid for $|m| \leq l$ and $1 \leq s \leq l$. In all other cases the harmonics are defined to be identically zero, except for $s=$ 0 , when $Z_{l}^{m} \equiv Y_{l}^{m}$. Note that, in fact, we do not need symmetrization on the far right-hand side because the tensors $Z$ and $X$ are traceless. All other objects in the basis can be obtained from products of $\gamma, \epsilon$, and the basis for tensors of order $s-2$. For example, the basis for threeindex tensors is given by $Z_{l a b c}^{m}, X_{l_{a b c}}^{m}$, and six independent combinations of $\gamma_{a b} Z_{l c}^{m}, \gamma_{a b} X_{l c}^{m}, \epsilon_{a b} Z_{l c}^{m}, \epsilon_{a b} X_{l c}^{m}$, and their index permutations. The general case results from 
the iteration of the relations (valid for $s \geq 2$ ):

$$
\begin{aligned}
& Z_{l a_{1} \ldots a_{s}: b}^{m}=Z_{l a_{1} \ldots a_{s} b}^{m}+\frac{(l+s)(l-s+1)}{2} \\
& \times\left[\frac{1}{2} \gamma_{\left(a_{1} a_{2}\right.} Z_{\left.l a_{3} \ldots a_{s}\right) b}^{m}-\gamma_{b\left(a_{1}\right.} Z_{\left.l a_{2} \ldots a_{s}\right)}^{m}\right], \\
& X_{l a_{1} \ldots a_{s}: b}^{m}=X_{l a_{1} \ldots a_{s} b}^{m}+\frac{(l+s)(l-s+1)}{2} \\
& \times\left[\frac{1}{2} \gamma_{\left(a_{1} a_{2}\right.} X_{l_{\left.a_{3} \ldots a_{s}\right) b}^{m}}-\gamma_{b\left(a_{1}\right.} X_{\left.l_{a_{2} \ldots a_{s}}\right)}\right] .
\end{aligned}
$$

Appendix $\mathrm{B}$ gives a different approach to expand the definitions (31) and (32).

Remembering the definitions of the scalars $Z_{l}^{m} \equiv Y_{l}^{m}$ and $X_{l}^{m} \equiv 0$, and those of the vectors $Z_{l a}^{m} \equiv Y_{l: a}^{m}$ and $X_{l a}^{m} \equiv S_{l a}^{m}$, we obtain the three remaining special cases:

$$
\begin{gathered}
Z_{l: a}^{m}=Z_{l a}^{m}, \\
Z_{l a: b}^{m}=Z_{l a b}^{m}-\frac{l(l+1)}{2} \gamma_{a b} Z_{l}^{m}, \\
X_{l a: b}^{m}=X_{l a b}^{m}-\frac{l(l+1)}{2} \epsilon_{a b} Z_{l}^{m} .
\end{gathered}
$$

Formulas (33)-(37) for the STF tensors $Z$ and $X$ constitute a complete set of simplification rules which allow us to express any derivative of a tensor harmonic field on the sphere in a unique canonical way. Note that $\epsilon_{a b}$ appears only in Eq. (37).

Finally, it is important to point out that all harmonics have a well-defined parity under inversion of axes. This is because the parity of $\gamma_{a b}, \epsilon_{a b}$, and the scalar harmonics $Y_{l}^{m}$ is $+1,-1$, and $(-1)^{l}$, respectively, and because taking covariant derivatives does not change the parity. It is usual to separate harmonics with momentum $l$ into two families: tensors $Z_{l a_{1} \ldots a_{s}}^{m}$ have parity $(-1)^{l}$ (these are said to be "polar" or of "even" polarity), and tensors $X_{l a_{1} \ldots a_{s}}^{m}$ have parity $(-1)^{l+1}$ ("axial" or "odd" polarity). One must not confuse parity and polarity: whereas all equations must have a well-defined parity at any order in perturbation theory, polarity is only useful in the first-order theory because products of harmonics couple the two polarities, as we will see.

\section{A formula for the product of tensor harmonics}

The product of two scalar harmonics can be expanded in terms of finite sums of scalar harmonics using ClebschGordan coefficients [39]:

$$
Y_{l^{\prime}}^{m^{\prime}} Y_{l}^{m}=\sum_{l^{\prime \prime}=\left|l^{\prime}-l\right|}^{l^{\prime}+l} E_{0 l^{\prime} m^{\prime} l^{\prime \prime}}^{0 l m} Y_{l^{\prime \prime}}^{m+m^{\prime}},
$$

where we have defined the symbol

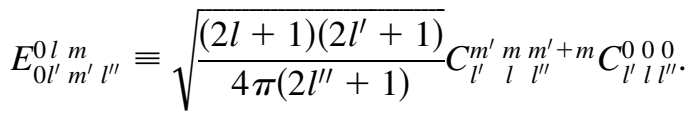

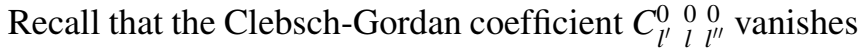
if $l^{\prime}+l+l^{\prime \prime}$ is odd. This fact guarantees that only scalars with parity $(-1)^{l^{\prime \prime}}=(-1)^{l^{\prime}+l}$ are present in the expansion.

In this subsection we will construct a generalization of Eq. (38) valid for any pair of tensor harmonics on the 2sphere. There are two main routes to find such a formula. The standard route, explored in Appendix $\mathrm{C}$ and followed by most books in quantum mechanics, is adapted to the 3dimensional Euclidean structure of $R^{3}$ and uses the socalled "pure-orbital" harmonics $\mathcal{O}_{l}^{j, m}{ }_{i_{1} \ldots i_{s}}$, which transform under a representation of "total angular momentum" $j$, with $|m| \leq j$, and whose Cartesian components are eigenfunctions of the "orbital angular momentum" operator (C3) with eigenvalue $l(l+1)$. This latter property becomes very useful when solving wave equations in a 3-dimensional setting. Unfortunately, these harmonics are not transverse to the radial direction, a fact that unnecessarily complicates the analysis of the radiation in the far region.

Here we will follow the second route, based on the socalled "pure-spin" harmonics $\mathcal{Y}_{l}^{ \pm s, m}{ }_{a_{1} \ldots a_{s}}$. They are adapted to the 2-sphere, and hence are transverse to the radial direction. Besides, they are closely related to the Wigner representation matrices of the rotation group, for which a product formula is well known. These harmonics can be defined in the following way.

Let us consider the unit sphere, with points described in a certain frame by coordinates $\left(\theta^{\prime}, \phi^{\prime}\right)$. A rotation $R$ of that frame assigns new coordinates $(\theta, \phi)$ to the same physical points, so that scalar fields $f$ transform as

$$
f^{\prime}(\theta, \phi)=f\left(\theta^{\prime}, \phi^{\prime}\right) \equiv D(R) f(\theta, \phi) .
$$

Since spherical harmonics provide an irreducible representation of the rotation group, their transformation rule must have the form

$$
\begin{aligned}
D(R) Y_{l}^{m}(\theta, \phi) & =Y_{l}^{m}\left(\theta^{\prime}, \phi^{\prime}\right) \\
& =\sum_{m^{\prime}=-l}^{l} \mathcal{D}_{m^{\prime} m}^{(l)}(\alpha, \beta, \gamma) Y_{l}^{m^{\prime}}(\theta, \phi),
\end{aligned}
$$

where $\alpha, \beta$, and $\gamma$ are the three Euler angles corresponding to the rotation $R$. Explicit formulas for the components of the matrices $\mathcal{D}_{m^{\prime} m}^{(l)}(\alpha, \beta, \gamma)$ have been computed by Wigner [39] and are reproduced in Appendix A. Most important for us, the product of any two of these matrices for the same rotation $R$ can be expanded using Clebsch- 
Gordan coefficients:

$$
\begin{aligned}
\mathcal{D}_{m_{1}^{\prime} m_{1}}^{\left(j_{1}\right)}(R) \mathcal{D}_{m_{2}^{\prime} m_{2}}^{\left(j_{2}\right)}(R)= & \sum_{j} C_{j_{1} j_{2} j}^{m_{1} m_{2} m_{1}+m_{2}} C_{j_{1} j_{2} j}^{m_{1}^{\prime} m_{2}^{\prime} m_{1}^{\prime}+m_{2}^{\prime}} \\
& \times \mathcal{D}_{m_{1}^{\prime}+m_{2}^{\prime}, m_{1}+m_{2}}^{(j)}(R) .
\end{aligned}
$$

Making use of these matrices, the spin-weighted scalar harmonics can be defined as [42]

$$
{ }_{s} Y_{l, m}(\theta, \phi) \equiv \sqrt{\frac{2 l+1}{4 \pi}} \mathcal{D}_{-s, m}^{(l)}(0, \theta, \phi) .
$$

In particular, ${ }_{0} Y_{l, m}=Y_{l}^{m}$.

STF tensors on the unit sphere can be constructed from the vectors $m^{a}$ and $\bar{m}^{a}$ defined in Eq. (20). Two independent STF tensors of rank $s$ are

$$
m^{a_{1}} \ldots m^{a_{s}} \text { and } \bar{m}^{a_{1}} \ldots \bar{m}^{a_{s}}
$$

because $\gamma_{a b} m^{a} m^{b}=0=\gamma_{a b} \bar{m}^{a} \bar{m}^{b}$. We then define the pure-spin tensor harmonics with $s \geq 0$ indices on $S^{2}$ as

$$
\begin{gathered}
\mathcal{Y}_{l a_{1} \ldots a_{s}}^{s, m} \equiv(-1)^{s} k(l, s) \mathcal{D}_{s, m}^{(l)}(0, \theta, \phi) m_{a_{1}} \ldots m_{a_{s}}, \\
\mathcal{Y}_{l}^{-s, m}{ }_{a_{1} \ldots a_{s}} \equiv k(l, s) \mathcal{D}_{-s, m}^{(l)}(0, \theta, \phi) \bar{m}_{a_{1}} \ldots \bar{m}_{a_{s}},
\end{gathered}
$$

with

$$
k(l, s)=\sqrt{\frac{(2 l+1)(l+s) !}{2^{s+2} \pi(l-s) !}} .
$$

The normalization factors are introduced so that the GS harmonics are (for $s \geq 1$ )

$$
\begin{gathered}
Z_{l a_{1} \ldots a_{s}}^{m}=\mathcal{Y}_{l a_{1} \ldots a_{s}}^{s, m}+\mathcal{Y}_{l}^{-s, m}{ }_{a_{1} \ldots a_{s}}, \\
-i X_{l a_{1} \ldots a_{s}}^{m}=\mathcal{Y}_{l}^{s, m}{ }_{a_{1} \ldots a_{s}}-\mathcal{Y}_{l}^{-s, m}{ }_{a_{1} \ldots a_{s}} .
\end{gathered}
$$

For the case $s=0$, one has $Z_{l}^{m}=\mathcal{Y}_{l}^{0, m}=Y_{l}^{m}$.

We can invert the previous relations to get (except for the special case $s=0$ )

$$
\begin{gathered}
\mathcal{Y}_{l a_{1} \ldots a_{s}}^{s, m}=\left(m_{a_{1}} \bar{m}^{b} Y_{l: b a_{2} \ldots a_{s}}^{m}\right)^{\mathrm{STF}}, \\
\mathcal{Y}_{l}^{-s, m}{ }_{a_{1} \ldots a_{s}}=\left(\bar{m}_{a_{1}} m^{b} Y_{l: b a_{2} \ldots a_{s}}^{m}\right)^{\mathrm{STF}} .
\end{gathered}
$$

Formula (42) provides the following product of pure-spin harmonics with the same sign:

$$
\begin{aligned}
\mathcal{Y}_{l^{\prime}}^{ \pm s^{\prime}, m^{\prime}} & \mathcal{Y}_{a_{1} \ldots a_{s^{\prime}}}^{ \pm s, m}{ }_{b_{1} \ldots b_{s}} \\
= & \sum_{l^{\prime \prime}=\left|l-l^{\prime}\right|}^{l^{\prime}+l} E_{ \pm s}^{ \pm s^{\prime}} l^{l^{\prime}} m^{\prime} \\
m^{\prime \prime} & \mathcal{Y}_{l^{\prime \prime}}^{ \pm\left(s^{\prime}+s\right), m^{\prime}+m}{ }_{a_{1} \ldots a_{s^{\prime}} b_{1} \ldots b_{s}},
\end{aligned}
$$

where we have introduced the real coefficients

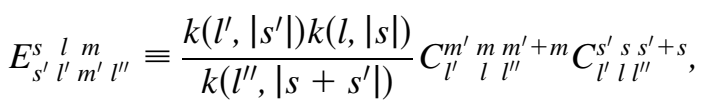

which generalize the coefficients (39). These inherit from the Clebsch-Gordan coefficients the symmetry properties

$$
\begin{gathered}
E_{-s l m}^{-s^{\prime} l^{\prime} m^{\prime}}=E_{s}^{s^{\prime} l^{\prime}-m^{\prime}}=(-1)^{l^{\prime}+l-l^{\prime \prime}} E_{s l}^{s^{\prime} l^{\prime} m^{\prime} l^{\prime \prime}}, \\
E_{s l m l^{\prime \prime}}^{s^{\prime} l^{\prime} m^{\prime}}=E_{s^{\prime} l^{\prime} m^{\prime} l^{\prime \prime}}^{s} .
\end{gathered}
$$

From the fact that $C_{l}^{m m} l_{l^{\prime \prime}}^{2 m}=0$ for odd $l^{\prime \prime}$, we also get that the $E$ coefficients vanish for odd $l^{\prime \prime}$ if $l=l^{\prime}$ and either $m=$ $m^{\prime}$ or $s=s^{\prime}$.

For the remaining products of pure-spin harmonics (those with opposite signs), we obtain (assuming e.g. that $s^{\prime} \geq s$ without loss of generality)

$$
\begin{aligned}
& \mathcal{Y}_{l^{\prime}}^{\mp s^{\prime}, m^{\prime}}{ }_{a_{1} \ldots a_{s^{\prime}}} \mathcal{Y}_{l}^{ \pm s, m}{ }_{b_{1} \ldots b_{s}} \\
& =\sum_{l^{\prime \prime}=\left|l-l^{\prime}\right|}^{l^{\prime}+l} E_{ \pm s}^{\mp s^{\prime} l^{\prime} m^{\prime} l^{\prime \prime}} \mathcal{Y}_{l^{\prime \prime}}^{\mp\left(s^{\prime}-s\right), m^{\prime}+m}{ }_{a_{s+1} \ldots a_{s^{\prime}}} T^{ \pm s}{ }_{a_{1} b_{1} \ldots a_{s} b_{s}},
\end{aligned}
$$

where the products

$$
T^{s}{ }_{a_{1} b_{1} \ldots a_{s} b_{s}} \equiv(-1)^{s} \bar{m}_{a_{1}} m_{b_{1}} \ldots \bar{m}_{a_{s}} m_{b_{s}},
$$

$$
T_{a_{1} b_{1} \ldots a_{s} b_{s}}^{-s} \equiv(-1)^{s} m_{a_{1}} \bar{m}_{b_{1}} \ldots m_{a_{s}} \bar{m}_{b_{s}}
$$

must be expanded using Eq. (21). We define $T^{0} \equiv 1$.

Adopting the notation ${ }^{( \pm)} \mathcal{Z} \equiv \mathcal{Y}^{s} \pm \mathcal{Y}^{-s}$ for all $s$ (where we have obviated the rest of the subindices and superindices), and introducing the tensors $\mathcal{T}^{ \pm} \equiv \frac{1}{2}\left(T^{-s} \pm\right.$ $T^{s}$ ) and the alternating $\operatorname{sign} \varepsilon \equiv(-1)^{l+l^{\prime}-l^{\prime \prime}}$, we hence arrive at the final formula (assuming again that $s^{\prime} \geq s$ ) valid for the product of any two generalized harmonics,

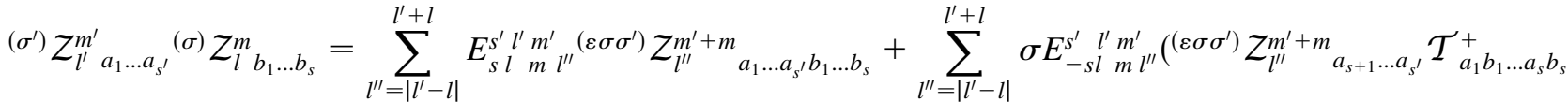

$$
\begin{aligned}
& \left.+{ }^{\left(-\varepsilon \sigma \sigma^{\prime}\right)} Z_{l^{\prime \prime}}^{m^{\prime}+m}{ }_{a_{s+1} \ldots a_{s^{\prime}}} \mathcal{T}_{a_{1} b_{1} \ldots a_{s} b_{s}}^{-}\right),
\end{aligned}
$$


which constitutes the main result of this section. The first sum in this formula is very simple [similar to that in Eq. (52)] and involves only harmonics with $s^{\prime}+s$ indices. The second sum involves harmonics with $s^{\prime}-s$ indices and has a more complicated structure in order to include the case of products with scalar harmonics.

$$
\begin{aligned}
& \Delta^{n}\left[g_{\mu \nu}\right] \equiv{ }^{\{n\}} h_{\mu \nu} \equiv \sum_{l, m}\left(\begin{array}{cc}
{ }^{\{n\}} H_{l A}^{m} Z_{l b}^{m}+{ }^{n}{ }^{m} H^{m}{ }_{l n} h_{l A}^{m} X_{l b}^{m} & { }^{\{n\}} H_{l A}^{m} Z_{l b b}^{m}+{ }^{\{n\}} h_{l A_{A}}^{m} X_{l b}^{m} K_{l}^{m} r^{2} \gamma_{a b} Z_{l}^{m}+{ }^{\{n\}} G_{l}^{m} r^{2} Z_{l a b}^{m}+{ }^{n n\}} h_{l}^{m} X_{l a b}^{m}
\end{array}\right),
\end{aligned}
$$

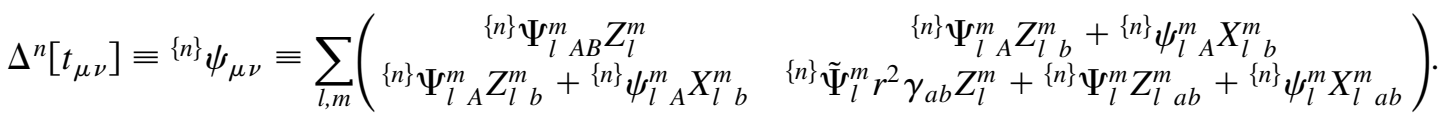

The polar (axial) components of the perturbations are denoted with uppercase (lowercase) letters. All indices have been displayed in this expression, forcing us to use up to five indices in the tensor perturbation ${ }^{\{n\}} H_{l}^{m}$. For $n=1$ these expansions in harmonics (and, in particular, the choice of factors $r^{2}$ ) reduce to those of Ref. [22], with some changes in the notation and the mentioned difference of normalization of the axial tensor $X_{l a b}^{m}$.

\section{B. Gauge dependence}

The perturbations (60) and (61) are not invariant under changes of gauge, i.e., under changes of the point-to-point identification between the perturbed and unperturbed spacetimes. Those changes can be thought of as diffeomorphisms on one of those manifolds. Sonego and Bruni [43] have given a complete description of the Taylor expansion of families of diffeomorphisms around the identity map. A given family is described by an infinite collection of vector fields ${ }^{\{n\}} \xi^{\mu}$-one at each order in the power series expansion-in terms of which the action of the diffeomorphisms can be expressed using Lie derivatives. For example, up to second order the result is

$$
\begin{gathered}
\overline{\Delta[T]}-\Delta[T]=\mathcal{L}_{\{1\}} T, \\
\overline{\Delta^{2}[T]}-\Delta^{2}[T]=\mathcal{L}_{\{2\}} T+2 \mathcal{L}_{\{1\} \xi} \Delta[T]+\mathcal{L}_{\{1\}}^{2} T,
\end{gathered}
$$

for any tensor field $T$, where the overline denotes a different choice of gauge. In particular, for the metric field,

$$
\begin{gathered}
\overline{{ }^{\{1\}} h_{\mu \nu}}-{ }^{\{1\}} h_{\mu \nu}=\mathcal{L}_{\{1\} \xi} g_{\mu \nu}, \\
\overline{\{2\} h_{\mu \nu}}-\{2\} h_{\mu \nu}=\mathcal{L}_{\{2\}} g_{\mu \nu}+2 \mathcal{L}_{\{1\} \xi}\{1\} h_{\mu \nu}+\mathcal{L}_{\{1\} \xi}^{2} g_{\mu \nu} .
\end{gathered}
$$

These formulas allow us to compute the perturbations in any desired gauge from their values in a particular one.
In principle, it may be possible to form gauge-invariant combinations of the perturbations at any desired order. For simplicity, nonetheless, here we will perform our calculations by imposing a particular choice of gauge: the generalization to higher orders $(n>1)$ of the RW gauge, namely,

$$
{ }^{\{n\}} H_{l A}^{m}=0, \quad{ }^{\{n\}} G_{l}^{m}=0, \quad{ }^{\{n\}} h_{l}^{m}=0 .
$$

This leads to a full metric $\tilde{g}_{\mu \nu}$ whose components $A b$ and $a b$ obey four local gauge conditions at all points,

$$
\tilde{g}_{A b: c} g^{b c}=0, \quad \tilde{g}_{a b}=\tilde{K} g_{a b},
$$

for some generic scalar field $\tilde{K}$ on $M^{4}$. To be more precise, the RW conditions select the point-to-point mapping between the perturbed and unperturbed spacetimes in such a way that the pullback $\tilde{g}$ of the perturbed metric into the background manifold obeys Eq. (67). Conversely, using these four conditions it is easy to see that it is always possible to impose the RW gauge at all perturbation orders in any family of metrics, at least at a local level, so that the gauge is well posed.

\section{Evolution equations}

The spherical background metric (60) satisfies the Einstein equations $G_{\mu \nu}=8 \pi t_{\mu \nu}$, which can be decomposed as [21]

$$
\begin{gathered}
G_{A B}=-2\left(v_{A \mid B}+v_{A} v_{B}\right)+g_{A B}\left(-\frac{1}{r^{2}}+2 v_{C} \mid C+3 v_{C} v^{C}\right) \\
=8 \pi t_{A B}, \\
G_{a}{ }^{a}=-{ }^{(2)} R+2 v_{A}^{\mid A}+2 v_{A} v^{A}=8 \pi Q
\end{gathered}
$$

The evolution of the first-order perturbations is schematically given by the six GS equations 


$$
\begin{gathered}
E_{A B}\left[{ }^{\{1\}} h_{l}^{m}\right]=8 \pi^{\{1\}} \Psi_{l A B}^{m}, \\
E_{A}\left[{ }^{\{1\}} h_{l}^{m}\right]=8 \pi^{\{1\}} \Psi_{l A}^{m}, \\
\tilde{E}\left[{ }^{\{1\}} h_{l}^{m}\right]=8 \pi^{\{1\}} \tilde{\Psi}_{l}^{m}, \\
E\left[{ }^{\{1\}} h_{l}^{m}\right]=8 \pi^{\{1\}} \Psi_{l}^{m}, \\
O_{A}\left[{ }^{\{1\}} h_{l}^{m}\right]=8 \pi\left(\{1\} \psi_{l A}^{m}-\frac{1}{2} Q^{\{1\}} h_{l A}^{m}\right), \\
O\left[{ }^{\{1\}} h_{l}^{m}\right]=8 \pi^{\{1\}} \psi_{l}^{m},
\end{gathered}
$$

where ${ }^{\{1\}} h_{l}^{m}$ represents the 10 first-order perturbations with labels $l$ and $m$, and the $E$ and $O$ differential operators are given in Appendix D. The $E$ operators contain only polar metric perturbations and the $O$ operators only axial ones.

The evolution of the second-order perturbations is dictated by the same equations, except for that now the lefthand side contains extra sources that are quadratic in the first-order perturbations:

$$
\begin{aligned}
& E_{A B}\left[{ }^{\{2\}} h_{l}^{m}\right]+\sum_{\bar{l}, \hat{l}} \sum_{\bar{m}, \hat{m}}{ }^{(\varepsilon)} S_{\bar{l}}^{\bar{m} \hat{l}{ }_{l} l A B}=8 \pi^{\{2\}} \Psi_{l A B}^{m}, \\
& E_{A}\left[{ }^{\{2\}} h_{l}^{m}\right]+\sum_{\bar{l}, \hat{l}} \sum_{\bar{m}, \hat{m}}{ }^{(\varepsilon)} S_{\bar{l} \hat{l} l A}^{\bar{m} \hat{m}_{l} m}=8 \pi^{\{2\}} \Psi_{l A}^{m}, \\
& \tilde{E}\left[{ }^{\{2\}} h_{l}^{m}\right]+\sum_{\bar{l}, \hat{l}} \sum_{\bar{m}, \hat{m}}{ }^{(\varepsilon)} \tilde{S}_{\bar{l}}^{\bar{m} \hat{m} m l}=8 \pi^{\{2\}} \tilde{\Psi}_{l}^{m}, \\
& E\left[{ }^{\{2\}} h_{l}^{m}\right]+\sum_{\bar{l}, \hat{l}} \sum_{\bar{m}, \hat{m}}{ }^{(\varepsilon)} S_{\bar{l}}^{\bar{m} \hat{l} l l}=8 \pi^{\{2\}} \Psi_{l}^{m},
\end{aligned}
$$

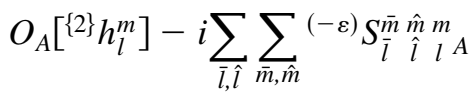

$$
\begin{aligned}
& =8 \pi\left({ }^{\{2\}} \psi_{l_{A}}^{m}-\frac{1}{2} Q^{\{2\}} h_{l_{A}}^{m}\right) \text {, } \\
& \left.O{ }^{[2\}} h_{l}^{m}\right]-i \sum_{\bar{l}, \hat{l}} \sum_{\bar{m}, \hat{m}}{ }^{(-\varepsilon)} S_{\bar{l}}^{\bar{m} \hat{m} m l}=8 \pi^{\{2\}} \psi_{l}^{m},
\end{aligned}
$$

with the usual restrictions on the values of $\bar{m}$ and $\hat{m}$, and with both $\bar{l}$ and $\hat{l}$ being independent and running over all nonnegative integers. The structure of the sources is rather peculiar, owing to the mixture of polarities that appears in the product of harmonics. This fact is encoded in the polarity sign $\sigma$ of the sources ${ }^{(\sigma)} S_{\bar{l}}^{\bar{m} \hat{m} m}$, which is always given in terms of the associated sign $\varepsilon \equiv(-1)^{\bar{l}+\hat{l}-l}$ and thus completely determined for each term of the sum, so that it cannot be chosen freely. Sources with polarity sign $\sigma=+1$ contain terms polar $\times$ polar and axial $\times$ axial with real coefficients. Sources with polarity $\operatorname{sign} \sigma=-1$ contain terms of the form polar $\times$ axial with purely imaginary coefficients. This form ensures an adequate behavior of the equations under complex conjugation (that we denote with the symbol *). In particular, using (54), we have for all sources and for all $\bar{l}, \hat{l}, l$

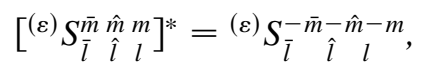

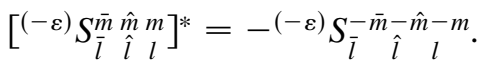

The sign $\varepsilon$ alternates when any of the $l$ labels change. Therefore, all equations have generically both types of sources.

On the other hand, we see that some pairs of equations share the sources: for example, Eqs. (79) and (81) alternate their sources ${ }^{(+)} S$ and ${ }^{(-)} S$ for particular sets of labels $\hat{l}, \bar{l}, l$. The same thing happens with the pair (77) and (80). The operators $E_{A B}$ and $\tilde{E}$, however, have their own pair of sources. As a result, we need to compute eight sources in total, instead of 12 .

Using the expansion (15) and the definition of the metric perturbations (60), we can expand the Einstein equations at second order assuming, without loss of generality, that there are only two first-order perturbations, but allowing these to be completely arbitrary, in particular, assigning arbitrary harmonic labels to them. From now on the coefficients and harmonic labels of those two perturbations will be denoted as $\hat{h}$ and $\bar{h}$, with all other perturbation amplitudes vanishing. In this way, we avoid dealing with sums that include (quadratic) couplings between an infinite number of first-order perturbations. The expansion contains many terms with products of tensor harmonics: we count 1275,972 , and 1347 source terms in $\Delta\left[G_{A B}\right], \Delta\left[G_{A b}\right]$, and $\Delta\left[G_{a b}\right]$, respectively (still at the $2+2$ abstract level, without any expansion in coordinate ranges). These products of harmonics must then be expanded using formula (59). We now analyze the sources separately.

The source of $\Delta\left[G_{A B}\right]$ contains products of harmonics of the form $\bar{Z}^{a b c} \hat{Z}_{a b c}, \bar{Z}^{a b} \hat{X}_{a b}$, etc. (harmonics with four indices do not appear in RW gauge). The final expression can be rearranged to arrive at the sources: 


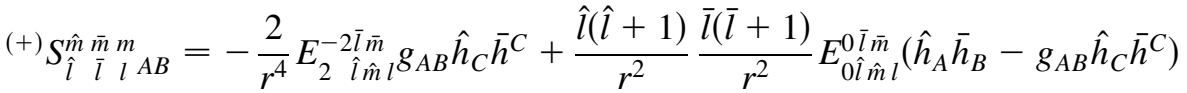

$$
\begin{aligned}
& +\frac{1}{r^{2}} E_{1 \hat{l} \hat{m} l}^{-1 \bar{l} l}\left\{4 \hat{h}^{C}\left(\bar{h}_{(A \mid B) C}-\bar{h}_{C \mid A B}+\bar{h}_{C} \boldsymbol{v}_{A \mid B}+2 \bar{h}_{C \mid(A} v_{B)}\right)+4 \hat{h}^{C}{ }_{\mid C} \bar{h}_{(A \mid B)}-2 \hat{h}^{C}{ }_{\mid A} \bar{h}_{C \mid B}-2 \bar{h}_{A}{ }^{\mid C} \hat{h}_{B \mid C}\right. \\
& +g_{A B}\left[2 \hat{h}^{(C \mid D)} \bar{h}_{(C \mid D)}-\hat{h}^{C} \bar{h}^{D}\left(12 \frac{r_{\mid C D}}{r}-4 v_{C} v_{D}\right)-2 r^{-4}\left(r^{2} \hat{h}^{C}\right)_{\mid C}\left(r^{2} \bar{h}^{D}\right)_{\mid D}\right. \\
& \left.\left.+\left(2^{(2)} R-4 \frac{\hat{l}^{2}+\hat{l}-1}{r^{2}}+\frac{4}{3} \frac{\left(r^{3}\right)^{\mid D}{ }_{D}}{r^{3}}\right) \hat{h}^{C} \bar{h}_{C}+\frac{4}{r^{2}} \epsilon^{C D} \hat{h}_{C}\left(r^{4} \bar{\Pi}\right)_{\mid D}-2 r^{4} \hat{\Pi} \bar{\Pi}+4 r^{2} \epsilon^{C D} \bar{h}_{C} v_{D} \hat{\Pi}\right]\right\} \\
& +\frac{1}{2 r^{2}} E_{1 \hat{l} \hat{l} \hat{m} l}^{-1 \bar{m}}\left[2 \hat{H}_{C}^{C} \bar{H}_{A B}-4 \bar{H}_{A C} \hat{H}_{B}^{C}+g_{A B}\left(4 \hat{K} \bar{K}+3 \hat{H}^{C D} \bar{H}_{C D}-\hat{H}_{C}^{C} \bar{H}^{D}{ }_{D}\right)\right] \\
& +E_{0 \hat{l} \hat{m} l}^{0 \bar{l} \bar{m}}\left\{-\frac{\hat{l}^{2}+\hat{l}+\bar{l}^{2}+\bar{l}-2}{r^{2}} \hat{H}_{A B} \bar{K}+\bar{H}^{C}{ }_{A B} \hat{K}_{\mid C}+\frac{2}{r^{3}} \hat{H}_{A B}\left(r^{3} \bar{K}_{\mid C}\right)^{\mid C}+(\hat{K} \bar{K})_{\mid A B}-\hat{K}_{\mid A} \bar{K}_{\mid B}+4 v_{(A} \hat{K}_{\mid B)} \bar{K}\right. \\
& +\frac{1}{2} \hat{H}_{C D(A} \bar{H}_{B)}^{C}{ }_{B}^{D}-\hat{H}^{C D}\left[r^{-2}\left(r^{2} \bar{H}_{C A B}\right)_{\mid D}-\bar{H}_{C D \mid B A}\right]-\frac{1}{2} \hat{H}_{D A B} \bar{H}_{C C}^{D C}+\left(\hat{H}_{A B}-g_{A B} \hat{H}_{F}^{F}\right) \\
& \times\left[\bar{H}_{C}^{C}{ }_{D}^{\mid D}-\bar{H}_{\mid C D}^{C D}+2 \bar{H}_{C \mid D}^{C} v^{D}-4 \bar{H}_{\mid C}^{C D} v_{D}-2 \bar{H}^{C D}\left(2 v_{C \mid D}+3 v_{C} v_{D}\right)+\bar{H}_{C}^{C}\left(\frac{2(2)}{2}-\frac{\bar{l}^{2}+\bar{l}}{r^{2}}\right)\right] \\
& +g_{A B}\left[\frac{\hat{l}^{2}+\hat{l}}{r^{2}}\left(\hat{H}_{C}^{C}+2 \hat{K}\right) \bar{K}-\bar{H}^{D C}{ }_{C} \hat{K}_{\mid D}-\frac{2}{r^{3}} \hat{H}^{C D}\left(r^{3} \bar{K}_{\mid C}\right)_{\mid D}-\frac{2}{r^{2}} \hat{K} \bar{K}-\frac{1}{r^{3}}\left[r^{3}(\hat{K} \bar{K})_{\mid C}\right]^{\mid C}\right. \\
& +\frac{3}{2} \hat{K}_{\mid C} \bar{K}^{\mid C}+2 \bar{H}^{F D E}\left[\left(\hat{H}_{F C} v^{C}-\hat{H}_{C}^{C} v_{F}\right) g_{D E}+v_{F} \hat{H}_{D E}\right]+\frac{1}{4} \hat{H}_{F C}{ }^{C} \bar{H}^{F D}{ }_{D} \\
& \left.\left.-\frac{1}{4} \hat{H}_{C D F} \bar{H}^{C D F}+\left(\hat{H}^{C D} \bar{H}_{C}{ }^{F}-\hat{H}_{C}^{C} \bar{H}^{D F}\right)\left[g_{D F}\left(\frac{\bar{l}^{2}+\bar{l}}{r^{2}}-\frac{{ }^{(2)} R}{2}\right)+2\left(2 v_{D \mid F}+3 v_{D} v_{F}\right)\right]\right]\right\} \text {, } \\
& { }^{(-)} S_{\hat{l} \bar{l} \bar{l} l A B}^{\hat{m} m}=\frac{2 i}{r^{2}} E_{1 \hat{l} \hat{m} l}^{-1 \bar{m} \bar{m}}\left\{\hat{H}_{A B} \bar{h}_{\mid C}^{C}+\hat{H}_{C}^{C} \bar{h}_{(A \mid B)}-2 \hat{H}_{(A}^{C} \bar{h}_{B) \mid C}+2\left(\hat{H}_{A B \mid C}-\hat{H}_{C(A \mid B)}\right) \bar{h}^{C}+g_{A B}\left[\hat{H}^{C D} \frac{1}{r^{2}}\left(r^{2} \bar{h}_{C}\right)_{\mid D}\right.\right. \\
& \left.\left.-\hat{H}_{C}^{C} \bar{h}_{\mid D}^{D}+2\left(\hat{H}_{D \mid C}^{C}-\hat{H}_{C \mid D}^{C}-\hat{K}_{\mid D}\right) \bar{h}^{D}\right]\right\} .
\end{aligned}
$$

We have employed that the sums in $\bar{l}$ and $\hat{l}$ are symmetric to simplify the form of the sources. Although each individual source ${ }^{(\sigma)} S_{\bar{l}}^{\bar{m} \hat{m} m}$ is not symmetric under the interchange $(\bar{l}, \bar{m}) \leftrightarrow(\hat{l}, \hat{m})$, their sum is symmetrized. We have also tried to simplify the expressions as much as possible by using the GS scalar $\Pi=\epsilon^{A B}\left(r^{-2} h_{A}\right)_{\mid B}$. In addition, we have defined $H_{A B C} \equiv H_{A B \mid C}+H_{A C \mid B}-H_{B C \mid A}$.

On the other hand, the source of $\Delta\left[G_{A b}\right]$ can be decomposed as

$$
\begin{aligned}
& \left.{ }^{(+)} S_{\hat{l} \bar{l} l A}^{\hat{m} \bar{l} l A}=\frac{2}{r^{2}} E_{-1 \hat{l} \hat{m} l}^{2} \bar{l} \bar{l} l\left(\hat{h}_{[A \mid B]}+\hat{h}_{B} \boldsymbol{v}_{A}\right) \bar{h}^{B}-\hat{h}^{B} \bar{h}_{(A \mid B)}\right\}+E_{0 \hat{l} \hat{m} l}^{1 \bar{l} \bar{m}}\left\{\frac{1}{2} \hat{H}_{B C \mid A} \bar{H}^{B C}+\hat{H}^{B C}\left(\bar{H}_{B C \mid A}-\bar{H}_{A B \mid C}-\bar{H}_{B C} \boldsymbol{v}_{A}\right)\right. \\
& +\frac{1}{2}\left(\hat{H}_{B \mid C}^{B}-2 \hat{H}_{C \mid B}^{B}\right) \bar{H}_{A}^{C}+(\hat{K} \bar{K})_{\mid A}+\frac{1}{2} \hat{K}_{\mid A} \bar{H}_{B}^{B}+\frac{\hat{l}^{2}+\hat{l}}{r^{2}}\left[3\left(\hat{h}_{[A \mid B]}+\hat{h}_{B} v_{A}\right) \bar{h}^{B}-\hat{h}^{B} \bar{h}_{(A \mid B)}\right. \\
& \left.\left.+r^{2} \hat{h}_{A}\left(r^{-2} \bar{h}^{B}\right)_{\mid B}\right]\right\} \\
& { }^{(-)} S_{\hat{l} \bar{l} \bar{l} l A}^{\hat{m} \bar{m}}=\frac{-i}{r^{2}} E_{2}^{-1 \bar{l} \bar{l} \bar{m} l}\left\{\hat{H}_{A B} \bar{h}^{B}+\hat{h}^{B} \bar{H}_{A B}\right\}+\frac{i}{2} E_{1 \hat{l} \hat{m} l}^{0 \bar{l} \bar{m}}\left\{\frac{\bar{l}^{2}+\bar{l}}{r^{2}}\left[-\hat{H}_{A B} \bar{h}^{B}+\hat{h}^{B} \bar{H}_{A B}+\left(\hat{H}_{B}^{B}-2 \hat{K}\right) \bar{h}_{A}-\hat{h}_{A}\left(\bar{H}^{B}{ }_{B}+2 \bar{K}\right)\right]\right. \\
& +2 \hat{h}_{\mid A}^{B} \bar{K}_{\mid B}-2 r^{-2}\left(r^{2} \hat{h}^{B}\right)_{\mid B} \bar{K}_{\mid A}-2 r^{-2} \hat{h}^{B}\left(r^{2} \bar{K}_{\mid B}\right)_{\mid A}+\frac{2}{r^{2}} \hat{h}_{A}\left(2 \tilde{E}\left[{ }^{\{1\}} h_{\bar{l}}^{\bar{m}}\right]+\bar{K}\left(r^{2(2)} R-\hat{l}^{2}-\hat{l}\right)+\left(r^{2} \bar{K}\right)^{\mid B}{ }_{B}\right) \\
& \left.+2 r^{2} \epsilon^{B C} \hat{\Pi} \bar{H}_{A B \mid C}-r^{2} \epsilon_{A B} \hat{\Pi}\left(\bar{H}_{C}^{C \mid B}-2 \bar{H}_{\mid C}^{B C}\right)+2 r^{-2} \epsilon_{A B}\left(r^{4} \hat{\Pi}\right)_{\mid C} \bar{H}^{B C}\right\} \text {. }
\end{aligned}
$$

Finally, the manipulations for $\Delta\left[G_{a b}\right]$ are more complicated, involving up to 3091 terms in some intermediate steps. The resulting expression can be organized in the following four sources: 


$$
\begin{aligned}
& { }^{(+)} \tilde{S}_{\hat{l} \bar{l} l}^{\hat{m} \bar{m} m}=\frac{1}{r^{2}} E_{-1 \hat{l} \hat{m} l}^{1} \overline{\bar{m}} \bar{l}\left\{\hat{H}^{A B} \bar{H}_{A B}-\frac{1}{2} \hat{H}_{A}^{A} \bar{H}^{B}{ }_{B}+2 \hat{h}^{A \mid B} \bar{h}_{A \mid B}-\frac{2}{r^{2}}\left(r \hat{h}^{A}\right)_{\mid A}\left(r \bar{h}^{B}\right)_{\mid B}\right. \\
& \left.+2 \hat{h}^{A} \bar{h}^{B}\left[2 R_{A B}+3 v_{A} v_{B}-g_{A B} \frac{\bar{l}^{2}+\bar{l}-1-2 r r^{\mid C} \mid C}{r^{2}}\right]+\frac{4}{r} \epsilon^{A B} \hat{h}_{A}\left(r^{3} \bar{\Pi}\right)_{\mid B}\right\} \\
& +\frac{1}{2} E_{0 \hat{l} \hat{m} l}^{0 \bar{m} l}\left\{-\frac{\hat{l}^{2}+\hat{l}}{r^{2}} \frac{\bar{l}^{2}+\bar{l}}{r^{2}} \hat{h}^{A} \bar{h}_{A}+2\left(\hat{H}_{A}^{A}-\hat{K}\right)\left(\bar{H}^{B C}{ }_{\mid B C}-\bar{H}_{B}^{B}{ }_{B}{ }_{C}\right)+\frac{1}{2} \hat{H}^{A B \mid C}\left(2 \bar{H}_{A C \mid B}-3 \bar{H}_{A B \mid C}\right)\right. \\
& +\frac{1}{2}\left(2 \hat{H}_{B \mid A}^{A}-\hat{H}_{A \mid B}^{A}\right)\left(2 \bar{H}^{C B}{ }_{\mid C}-\bar{H}_{C}^{C}{ }_{C}^{\mid B}\right)+\hat{H}^{A B}\left[\bar{H}_{A B}\left(\frac{\bar{l}^{2}+\bar{l}}{r^{2}}-{ }^{(2)} R\right)-2 v_{B} \bar{H}_{C \mid A}^{C}+4\left(\bar{H}_{A C} v^{C}\right)_{\mid B}\right. \\
& \left.+\frac{4}{r}\left(r \bar{H}_{A C}\right)^{\mid C} v_{B}-2 \bar{H}_{A B \mid C} v^{C}\right]-2\left[\hat{H}^{A B} r^{-2}\left(r^{2} \bar{K}\right)_{\mid B}\right]_{\mid A}+\hat{H}_{A \mid B}^{A} r^{-2}\left(r^{2} \bar{K}\right)^{\mid B}+{ }^{(2)} R \bar{H}_{A}^{A} \hat{K}-4 \hat{H}^{A B} \bar{K} v_{A} v_{B} \\
& \left.-\hat{K}^{\mid A} \bar{K}_{\mid A}\right\} \\
& \left.{ }^{(-)} \tilde{S}_{\hat{l} \bar{l} \bar{m} l}^{\hat{m} \bar{m} m}=\frac{2 i}{r^{2}} E_{-1 \hat{l} \hat{m} l}^{1} \overline{\bar{l}} \bar{m}\left(g^{A B} \hat{H}_{C}^{C}-\hat{H}^{A B}\right) \bar{h}_{A \mid B}+\left(\hat{K}_{\mid B}+4 \hat{H}_{[A \mid B]}^{A}-\hat{H}_{A}^{A} \boldsymbol{v}_{B}\right) \bar{h}^{B}\right\}, \\
& { }^{(+)} S_{\hat{l} \hat{\bar{l}} \bar{l} l}^{\hat{m} \bar{m} m}=E_{1 \hat{l} \hat{l} \hat{m} l}^{1 \bar{l} \bar{m}}\left\{\frac{1}{2} \hat{H}^{A B} \bar{H}_{A B}+\hat{H}^{A}{ }_{A} \bar{K}+r^{4} \hat{\Pi} \bar{\Pi}-2 \frac{\hat{l}^{2}+\hat{l}-1}{r^{2}} \hat{h}^{A} \bar{h}_{A}\right\}+E_{0 \hat{l} \hat{m} l}^{2 \bar{l} \bar{m}} \hat{H}^{A B} \bar{H}_{A B}, \\
& { }^{(-)} S_{\hat{l} \bar{l} l}^{\hat{m} \bar{l} l}=-2 i E_{1 \hat{l} \hat{m} l}^{1 \bar{l} \bar{m}}\left(\hat{K} \bar{h}^{A}\right)_{\mid A}-i E_{0 \hat{l} \hat{m} l}^{2 \bar{l} \bar{m}}\left\{2\left(\hat{H}^{A B} \bar{h}_{A}\right)_{\mid B}-\hat{H}^{A}{ }_{A \mid B} \bar{h}^{B}\right\} .
\end{aligned}
$$

In spite of the obvious increase of complexity from the first-order to the second-order equations, we want to stress that the final expressions given above are still manageable and fully general, except for the choice of RW gauge. They can be particularized to the case of any spherical background, dynamical or not, containing any type of matter, and expressed in any kind of background coordinates (polar-radial, null, comoving, etc.)

In situations with just a single first-order perturbation, we will have $\hat{l}=\bar{l}$ and $\hat{m}=\bar{m}$. In these circumstances, the $E$ coefficients vanish for odd $l$. This implies that $\hat{l}+\bar{l}-l$ is always even and therefore the sources ${ }^{(-)} S$ are never excited in the polar equations (76)-(79); neither are the sources ${ }^{(+)} S$ in the axial equations (80) and (81). In particular, ${ }^{(-)} S_{A B}$ and ${ }^{(-)} \tilde{S}$ are never excited. This has been the case encountered in many previous investigations in second-order perturbation theory. For instance, for the perturbations of a slowly rotating star, a single axial $l=$ 1 mode has been assumed [15], and the studies of the closelimit black hole collisions have considered a single polar $l=2$ first-order perturbative mode [14]. It will be very interesting to discuss what kind of interactions between modes are excited through the other types of sources, an issue that we plan to analyze in future investigations.

\section{Energy-momentum conservation}

A complete set of evolution equations is obtained only after specifying the particular type of matter content of the system (including as such the vacuum). Some simple systems like scalar fields or perfect fluids are completely defined dynamically by energy-momentum conservation, but this is not the case in general. However, we can generally analyze the consequences of perturbing the matter conservation equations, as well as use this analysis as a check of the perturbed Bianchi identities, and hence as a consistency check of the sources given in the previous subsection.

Like every object in perturbation theory, the energymomentum conservation law can be expanded into a hierarchy of linear equations, all sharing the principal part,

$$
\Delta^{n}\left[T_{\mu \nu}^{; \nu}\right]=0 .
$$

At zeroth order we use the decomposition (18) provided by the background, which leads to a nontrivial relation

$$
Q v_{A}=\frac{1}{r^{2}}\left(r^{2} t_{A B}\right)^{\mid B} \text {. }
$$

Given its vectorial character, the energy-momentum conservation equation can be decomposed into three geometric parts at higher orders: a vector equation in the polar sector and two scalar (one polar and one axial) equations. At first order, those equations can be written in compact notation as

$$
\begin{gathered}
L_{A}\left[{ }^{\{1\}} \psi_{l}^{m},{ }^{\{1\}} h_{l}^{m}\right]=0, \\
L\left[{ }^{\{1\}} \psi_{l}^{m},{ }^{\{1\}} h_{l}^{m}\right]=0, \\
\tilde{L}\left[{ }^{\{1\}} \psi_{l}^{m},{ }^{\{1\}} h_{l}^{m}\right]=0 .
\end{gathered}
$$

The operators $L_{A}, L$, and $\tilde{L}$ are defined in Appendix D. 
The second-order perturbation adopts the same form with additional quadratic sources:

$$
\begin{aligned}
& \left.L_{A}{ }^{[2\}} \psi_{l}^{m},{ }^{\{2\}} h_{l}^{m}\right]+\sum_{\bar{l}, \hat{l}} \sum_{\bar{m}, \hat{m}}{ }^{(\varepsilon)} I_{\bar{l} \hat{m} \hat{l} l A}^{\hat{m} m}=0, \\
& L\left[{ }^{\{2\}} \psi_{l}^{m},{ }^{\{2\}} h_{l}^{m}\right]+\sum_{\bar{l}, \hat{l}} \sum_{\bar{m}, \hat{m}}{ }^{(\varepsilon)} I_{\bar{l}}^{\bar{m} \hat{l} \hat{l} m}=0,
\end{aligned}
$$

$$
\tilde{L}\left[{ }^{\{2\}} \psi_{l}^{m},{ }^{\{2\}} h_{l}^{m}\right]-i \sum_{\bar{l}, \hat{l}} \sum_{\bar{m}, \hat{m}}{ }^{(-\varepsilon)} I_{\bar{l}}^{\bar{m}} \hat{m} m=0 .
$$

Such sources can be computed starting from Eq. (92), decomposing it using formulas (60) and (61), and finally applying the tools that we have developed to deal with products of harmonics. The result, with the same notation used for the sources of the main equations, is

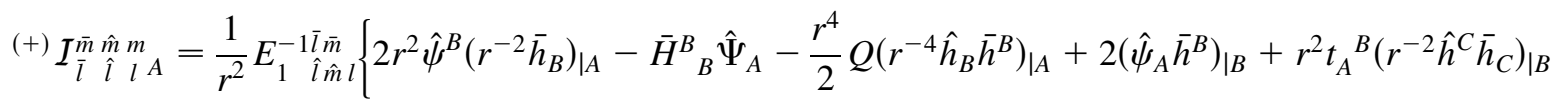

$$
\begin{aligned}
& \left.-2\left(\hat{h}^{C} \bar{h}^{B} t_{A B}\right)_{\mid C}-r^{2} t_{B C}\left(r^{-2} \hat{h}^{B} \bar{h}^{C}\right)_{\mid A}\right\}+\frac{1}{2} E_{0 \hat{l} \hat{m} l}^{0 \bar{l} \bar{l} l}\left\{\frac{2 \hat{l}(\hat{l}+1)}{r^{2}} \bar{K} \hat{\Psi}_{A}+2 \bar{\Psi}_{A}{ }^{B} \hat{K}_{\mid B}+2 Q r \bar{K}\left(r^{-1} \hat{K}\right)_{\mid A}\right. \\
& -2 \hat{K} \bar{K}^{\mid B} t_{A B}-2 r^{2} \overline{\tilde{\Psi}}\left(r^{-2} \hat{K}\right)_{\mid A}-2 \hat{H}^{B C} t_{A B} \bar{K}_{\mid C}-\hat{\Psi}^{B C} \bar{H}_{B C \mid A}+\hat{\Psi}_{A}^{B} \bar{H}_{C \mid B}^{C}-\frac{2}{r^{2}}\left(r^{2} \hat{\Psi}_{A B} \bar{H}^{B C}\right)_{\mid C} \\
& \left.+\hat{H}^{B C}\left[4 \bar{H}_{C}{ }^{D}{ }_{\mid(A} t_{D) B}-\bar{H}^{D}{ }_{D \mid C} t_{A B}-\bar{H}_{B C \mid D} t_{A}{ }^{D}+\frac{2}{r^{2}}\left(r^{2} \bar{H}_{B D} t_{A}{ }^{D}\right)_{\mid C}\right]\right\}, \\
& { }^{(-)} I_{\bar{l} \hat{\bar{l}} \hat{l} l_{l A}}=-\frac{i}{r^{2}} E_{1 \hat{l} \hat{l} \hat{m} l}^{-1 \bar{m}}\left\{\bar{H}^{B}{ }_{B} \hat{\psi}_{A}+\left(2 \bar{H}^{B C} \hat{h}_{C}-\bar{H}^{C}{ }_{C} \hat{h}^{B}\right) t_{A B}-2 \hat{h}^{B} \bar{\Psi}_{A B}-2 r^{2} \bar{\Psi}^{B}\left(r^{-2} \hat{h}_{B}\right)_{\mid A}-2\left(\bar{\Psi}_{A} \hat{h}^{B}\right)_{\mid B}\right\}, \\
& { }^{(+)} I_{\bar{l} \hat{l} \hat{l} l}^{\bar{m} l}=\frac{1}{r^{2}} E_{2 \hat{l} \hat{m} l}^{-1 \bar{m} \bar{m}}\left\{2 \bar{h}_{A} \hat{\psi}^{A}+2 \bar{\psi}_{A} \hat{h}^{A}+2\left(\hat{\psi} \bar{h}^{A}\right)_{\mid A}-\hat{\Psi} \bar{H}_{A}^{A}-Q \hat{h}^{A} \bar{h}_{A}-2 \hat{h}^{A} \bar{h}^{B} t_{A B}\right\} \\
& +\frac{1}{r^{2}} E_{0 \hat{l} \hat{m} l}^{1 \bar{l} \bar{l}}\left\{\hat{l}(\hat{l}+1)\left[\bar{\psi}_{A} \hat{h}^{A}-\hat{\psi}_{A} \bar{h}^{A}-\bar{h}^{A} \hat{h}^{B} t_{A B}+\frac{Q}{2} \bar{h}^{A} \hat{h}_{A}\right]+(\bar{l}-1)(\bar{l}+2) \hat{K} \bar{\Psi}-2\left(r^{2} \bar{\Psi}_{A} \hat{H}^{A B}\right)_{\mid B}\right. \\
& +2 r^{2}\left[\bar{\Psi}^{A} \hat{K}_{\mid A}-\hat{\tilde{\Psi}} \bar{K}-\overline{\tilde{\Psi}} \hat{K}+Q \hat{K} \bar{K}+\frac{1}{2} \bar{\Psi}^{B} \hat{H}_{A \mid B}^{A}\right] \\
& \left.+r^{2} \bar{H}^{A B}\left[2 \hat{H}_{B C} t_{A}^{C}-\hat{\Psi}_{A B}-\frac{Q}{2} \hat{H}_{A B}+g_{A B}\left(\hat{\tilde{\Psi}}-\frac{Q}{2} \hat{K}\right)\right]\right\}, \\
& { }^{(-)} I_{\bar{l} \hat{m} \hat{l} l}^{\bar{l} l}=\frac{i}{r^{2}} E_{2 \hat{l} \hat{m} l}^{-1 \bar{l} \bar{m}}\left\{2 \hat{h}_{A} \bar{\Psi}^{A}-2 \bar{h}_{A} \hat{\Psi}^{A}-\hat{\psi} \bar{H}_{A}^{A}-2\left(\hat{\Psi} \bar{h}^{A}\right)_{\mid A}\right\}+\frac{i}{r^{2}} E_{0 \hat{l} \hat{m} l}^{1 \bar{l} \bar{l}}\left\{\hat{l}(\hat{l}+1)\left(\bar{h}_{A} \hat{\Psi}^{A}-\hat{h}_{A} \bar{\Psi}^{A}\right)\right. \\
& +(\bar{l}-1)(\bar{l}+2) \bar{\psi} \hat{K}-2\left(r^{2} \bar{\psi}_{A} \hat{H}^{A B}\right)_{\mid B}-2\left(r^{2} \hat{\tilde{\Psi}} \bar{h}^{A}\right)_{\mid A}+2 r^{2} \bar{\psi}^{A} \hat{K}_{\mid A}+\hat{K}\left(Q r^{2} \bar{h}^{A}\right)_{\mid A}+\left(Q r^{2} \hat{H}^{A B} \bar{h}_{B}\right)_{\mid A} \\
& \left.+r^{2} \hat{H}_{A \mid B}^{A}\left(\bar{\psi}^{B}-\frac{Q}{2} \bar{h}^{B}\right)\right\} \text {. }
\end{aligned}
$$

\section{CONCLUSIONS}

Perturbation theory has been highly successful in general relativity and nowadays still plays a relevant role in the simulations of the dynamics of many physical and astrophysical systems. On the one hand, it allows us to interpret certain dynamical processes as the evolution of a perturbative mode of a simpler process, or as the interaction among several of such modes. On the other hand, using perturbation theory, we can evolve systems in which very different physical phenomena are happening simultaneously with so distinct space/time scales or amplitudes that a numerical simulation would fail to follow them all with a satisfactory precision. Even so, one must be aware of the problem of linearization stability when perturbing a given spacetime in general relativity; namely, it may happen that a perturba- tively constructed spacetime with parameter $\epsilon$ does not correspond to an exact family of solutions $g_{\mu \nu}(\epsilon)$ of the Einstein equations. This problem becomes more important when perturbing highly symmetric backgrounds [44].

However, going beyond first-order perturbation theory has not been feasible until very recently except in very specific situations. The present work proposes a systematic approach to high-order perturbation theory in general relativity, based on the combination of a good choice of the theoretical formalism employed for the description of the problem (implementing symmetry reductions, covariant notation, and other nice features) and the intensive use of abstract computer algebra to manipulate the enormous expressions that unavoidably appear in this field.

We have first given a number of formulas which permit us to compute very efficiently and at any order the pertur- 
bation of all relevant curvature tensors in general relativity, and have implemented them in the MATHEMATICA package XPERT. These formulas could be used in very different areas of gravitational physics, including theories that depart from standard general relativity (like in the case of models with extra dimensions, curvature corrections, or in braneworld scenarios).

We have then generalized to higher orders the wellknown GS formalism for nonspherical first-order perturbations of a spherical spacetime. This formalism is considered to be optimal for the perturbative study of a number of astrophysical scenarios of interest, except for the possible construction of master scalars and equations referred to in the Introduction. The generalization of the GS formalism put forward here will make the perturbative analysis even more powerful, leading to more precise results and allowing us to describe interactions between different modes.

With this purpose, we have constructed a generalization of the GS harmonics, which turn out to be very closely related to the Wigner rotation matrices (spin-weighted harmonics in the general relativity community). We have also obtained a general formula for the product of any pair of them, and implemented all these results in another package called HARMONICS.

In addition, we have computed all the equations of the generalized GS formalism at second order (including those of energy-momentum conservation) and simplified them to a form manageable enough as to allow us to write them down in this paper. These equations are completely general except for the restriction to a spherical background: they can be used with any background, dynamical or not, they can be coupled to any matter model, and they have been given in covariant form, so that any coordinate system can be used on the background manifold. We have presented the results in RW gauge, which is well posed at all perturbative orders. The original GS formalism also employs gauge-invariant variables, and we are currently working in this direction, with the aim of determining the gauge invariants at second order in full generality.

The second-order equations are essentially the same as the first-order equations, but they also include complicated quadratic sources. We have disentangled the structure of these sources and shown that, in previous investigations considering just a single first-order perturbative mode, many of such sources were not excited. We plan to study the role of these new sources in future work.

Let us conclude remarking that, once the gauge invariants are determined, the formalism and our computeralgebra tools implementing it will be ready to be applied to problems of astrophysical or conceptual relevance, such as the emission of gravitational radiation in the collapse of a rotating star, or the coupling of perturbative modes of a critical spacetime.

\section{ACKNOWLEDGMENTS}

D. B. acknowledges financial support from the FPI program of the Regional Government of Madrid. J. M. M.-G. acknowledges the financial aid provided by the I3P framework of CSIC and the European Social Fund. This work was also supported by the Spanish MEC Project No. FIS2004-01912 and No. FIS2005-05736-C03-02.

\section{APPENDIX A: SPHERICAL FUNCTIONS}

Several conventions are employed in the literature for the special functions used in the theory of representations of the 3-dimensional rotation group. Here we mostly follow the conventions of Edmonds [39].

The spherical harmonics $Y_{l}^{m}(\theta, \phi)$ are

$$
Y_{l}^{m}(\theta, \phi) \equiv \sqrt{\frac{(2 l+1)(l-m) !}{4 \pi(l+m) !}} P_{l}^{m}(\cos \theta) e^{i m \phi}
$$

where $P_{l}^{m}$ is the associated Legendre function

$$
P_{l}^{m}(x) \equiv \frac{(-1)^{m}}{2^{l} l !}\left(1-x^{2}\right)^{m / 2} \frac{d^{l+m}}{d x^{l+m}}\left(x^{2}-1\right)^{l} .
$$

The MATHEMATICA functions SphericalHarmonicY and LegendreP are indeed those defined above.

For a rotation of the reference frame described by the Euler angles $(\alpha, \beta, \gamma)$, the convention adopted for the unitary matrix in a representation $\mathcal{D}^{(l)}$ of $S U(2)$ is

$$
\mathcal{D}_{m^{\prime} m}^{(l)}(\alpha, \beta, \gamma)=e^{i m^{\prime} \alpha} d_{m^{\prime} m}^{(l)}(\beta) e^{i m \gamma} .
$$

The $\beta$ transformation is given by

$$
d_{m^{\prime} m}^{(l)}(\beta)=\sum_{\sigma} \frac{(-1)^{l-m^{\prime}-\sigma} \sqrt{(l+m) !(l-m) !\left(l+m^{\prime}\right) !\left(l-m^{\prime}\right) !}}{\left(l-m^{\prime}-\sigma\right) !(l-m-\sigma) !\left(m^{\prime}+m+\sigma\right) ! \sigma !}\left(\sin \frac{\beta}{2}\right)^{2 l-m^{\prime}-m-2 \sigma}\left(\cos \frac{\beta}{2}\right)^{m+m^{\prime}+2 \sigma}
$$

where the sum ranges over those integers $\sigma$ for which the arguments of the factorials are all nonnegative.

\section{APPENDIX B: SYMMETRIC TRACE-FREE TENSORS}

Given any tensor $T_{i_{1} \ldots i_{l}}$ over a vector space of dimension $d$ with a metric $g_{i j}$, we construct its STF part as 


$$
\left[T_{i_{1} \ldots i_{l}}\right]^{\mathrm{STF}} \equiv \sum_{m=0}^{[l / 2]} a_{l, d}^{(m)} g_{\left(i_{1} i_{2}\right.} \ldots g_{i_{2 m-1} i_{2 m}} S_{\left.i_{2 m+1} \ldots i_{l}\right)}{ }^{j_{1}}{ }_{j_{1} \ldots} j_{j_{m}}
$$

with $S_{i_{1} \ldots i_{l}} \equiv T_{\left(i_{1} \ldots i_{l}\right)}$ and $[l / 2]$ the integer part of $l / 2$. The coefficients of the expansion are determined by the tracefree condition, and are given by

$$
a_{l, d}^{(m)}=\frac{l !}{(-4)^{m} m !(l-2 m) !} \frac{\Gamma[l+d / 2-1-m]}{\Gamma[l+d / 2-1]} .
$$

In our case, $d=2$ for the unit sphere. These formulas allow us to compute any of the $Z_{l a_{1} \ldots a_{s}}^{m}$ in terms of the derivatives $Y_{l}^{m}: a_{1} \ldots a_{s}$ or vice versa. Note that derivatives of $Y_{l}^{m}$ with indices sorted differently are not equal, but can be transformed into a term with the desired order of indices plus terms with a lower number of derivatives.

\section{APPENDIX C: PURE-ORBITAL HARMONICS}

In this appendix, we briefly discuss the formalism of pure-orbital tensor harmonics and its relation with the pure-spin harmonics. First, one constructs pure-orbital vector harmonics by composing scalar harmonics of angular momentum $l$ with a set of 3-dimensional vectors $t^{m}{ }_{i}$ which transform under a representation of spin 1 [40]:

$$
\mathcal{O}_{l}^{j, m}{ }_{i} \equiv \sum_{m^{\prime}=-1}^{+1} C_{l}^{m-m^{\prime} m_{1}^{\prime} m} Y_{l}^{m-m^{\prime}} t^{m^{\prime}}
$$

with $j=l-1, l, l+1$, and $|m| \leq j$. The vectors $t^{m}{ }_{i}$ are defined in terms of a fixed orthonormal Cartesian basis:

$$
t^{ \pm 1}{ }_{i}=\frac{\mp e_{x i}-i e_{y i}}{\sqrt{2}}, \quad t^{0}{ }_{i}=e_{z i}
$$

(The index $i$ is an abstract index on the manifold $R^{3}$ with a Euclidean metric, in which $S^{2}$ is embedded.) These vector harmonics $\mathcal{O}_{l}^{j, m}{ }_{i}$ transform under a representation of total angular momentum $j$ and their Cartesian components are eigenfunctions, with eigenvalue $l(l+1)$, of the $S^{2}$ Laplacian (also called orbital angular momentum [41])

$$
L^{2} \equiv-r^{2} \vec{\nabla}^{2}+\partial_{r}\left(r^{2} \partial_{r}\right)=-\gamma^{a b} \nabla_{a} \nabla_{b} .
$$

Pure-orbital vector harmonics are, however, not transverse to the radial direction,

$$
\hat{r}_{i} \mathcal{O}_{l}^{j, m}{ }_{i}=-C_{j 1 l}^{000} Y_{j}^{m} .
$$

Therefore, one must take certain linear combinations canceling their radial contribution to get the GS harmonics.

Pure-orbital bases for higher-rank tensors can be constructed recursively from the above vector basis. The basis for STF tensors with $s$ indices and well-defined spin $s$ can be built by composition of the bases with $s^{\prime}$ and $s-s^{\prime}$ indices (with any $0<s^{\prime}<s$ ) as follows:

$$
t_{i_{1} \ldots i_{s}}^{m}=\sum_{m^{\prime}=-s^{\prime}}^{s^{\prime}} C_{s^{\prime} s-s^{\prime} s}^{m^{\prime} m-m^{\prime} m} t_{\left(i_{1} \ldots i_{s^{\prime}}\right.}^{m^{\prime}} t_{\left.s_{s^{\prime}+1} \ldots i_{s}\right)}^{m-m^{\prime}}
$$

From this, we construct orbital harmonics with $s$ indices:

$$
\mathcal{O}_{l}^{j, m} i_{1} \ldots i_{s} \equiv \sum_{m^{\prime}=-s}^{+s} C_{l}^{m-m^{\prime} m^{\prime} m} Y_{l}^{m-m^{\prime}} t^{m^{\prime}}{ }_{i_{1} \ldots i_{s}},
$$

which are normalized so that

$$
\int d \Omega\left(\mathcal{O}_{l}^{j, m}{ }_{i_{1} \ldots i_{s}}\right)^{*} \mathcal{O}_{l^{\prime}}^{j^{\prime}, m^{\prime}}{ }_{i_{1} \ldots i_{s}}=\delta_{l l^{\prime}} \delta_{j j^{\prime}} \delta_{m m^{\prime}} .
$$

The symbol $*$ denotes complex conjugation.

One can obtain the following multiplication rule by using Eq. (38) and the formulas available in the literature for the composition of three angular momenta [40]:

$$
Y_{l^{\prime}}^{m^{\prime}} \mathcal{O}_{l}^{j, m}{ }_{i_{1} \ldots i_{s}}=\sum_{l^{\prime \prime}=\left|l^{\prime}-l\right|}^{l^{\prime}+l} \sqrt{\frac{(2 l+1)\left(2 l^{\prime}+1\right)(2 j+1)}{4 \pi}} C_{l l^{\prime} l^{\prime \prime}}^{000} \sum_{j^{\prime}=l^{\prime \prime}-l}^{l^{\prime \prime}+l} W\left(s, j, l^{\prime \prime}, l^{\prime} ; l, j^{\prime}\right) C_{j l^{\prime}}^{m m^{\prime} m+m^{\prime}} \underset{j^{\prime}}{j^{\prime}, m+m^{\prime}}{ }_{i_{1} \ldots i_{s}}^{,}
$$

where $W$ is the Racah coefficient [39]. From this formula it is possible to compute the product of any two orbital harmonics using the Leibnitz rule, but here we prefer to show their connection with the pure-spin harmonics, which obey a much simpler multiplication formula.

The radial component of the orbital harmonics is

$$
\begin{aligned}
& \frac{\hat{r}_{i_{s}}^{i} \mathcal{O}_{l}^{j, m}{ }_{i_{1} \ldots i_{s-1} i_{s}}}{\sqrt{(2 s+1)(2 l+1)}} \\
& \quad=\sum_{l^{\prime}} C_{l 1 l^{\prime}}^{000} W\left(s-1,1, j, l ; s, l^{\prime}\right) \mathcal{O}_{l^{\prime}}^{j, m}{ }_{i_{1} \ldots i_{s-1}}
\end{aligned}
$$

which is a sum with only two contributions, $l^{\prime}=l \pm 1$.
Pure-spin harmonic tensors, with indices on $S^{2}$, can be obtained as linear combinations of the pure-orbital ones:

$$
\mathcal{Y}_{j}^{s, m} a_{a_{1} \ldots a_{s}}=\sum_{l=j-s}^{j+s} \sqrt{2 l+1} A_{j, s}^{l} \mathcal{O}_{l}^{j, m} a_{1} \ldots a_{s} .
$$

Asking them to be orthogonal to the radial direction, we find from Eq. (C9) that the coefficients of this expansion obey the "second-order" recursion relation

$$
A_{j, s}^{l+1}=f(l, j, s) f(l-1, j, s) A_{j, s}^{l-1},
$$




$$
f(l, j, s)=\sqrt{\frac{(s-l+j)(l+j+1-s)}{(s+l-j+1)(l+j+2+s)}} .
$$

Since the odd- $l$ coefficients decouple from the even- $l$ ones, there are two independent solutions. Fixing a normalization constant and employing definition (47), we get

$$
A_{j, s}^{(\mp) l}=C_{j}^{ \pm s \mp s 0} \frac{k(j, s)}{\sqrt{2 l+1}} \sqrt{\frac{4 \pi}{2 j+1}},
$$

which define, respectively, the harmonics $\mathcal{Y}_{j}^{ \pm s, m}{ }_{a_{1} \ldots a_{s}}$.

Finally, using formulas (42) and (C6), and the relation (43) (with $s=0$ ) between rotation matrices and spherical harmonics, one can express the pure-spin harmonics as

$$
\begin{aligned}
\mathcal{Y}_{j}^{ \pm s, m}{ }_{a_{1} \ldots a_{s}}= & k(l, s) \mathcal{D}_{ \pm s, m}^{(l)}(0, \theta, \phi) \\
& \times \sum_{m^{\prime}=-s}^{s}\left[\mathcal{D}_{ \pm s, m^{\prime}}^{(s)}(0, \theta, \phi)\right]^{*} t^{m^{\prime}}{ }_{a_{1} \ldots a_{s}} .
\end{aligned}
$$

\section{APPENDIX D: GS OPERATORS}

For the sake of completeness, we include in this appendix the metric part of the GS equations at first perturbative order and in RW gauge. This is also the linear part of the perturbation equations at any order. As it is usually done at first order, we obviate here the labels $l, m$, and $n$ of the perturbations on $M^{2}$.

$$
\begin{aligned}
& E_{A B}\left[{ }^{\{n\}} h_{l}^{m}\right] \equiv\left[\frac{(l-1)(l+2)}{2 r^{2}}+3 v_{C} v^{C}+2 v_{\mid C}^{C}\right] H_{A B}+v_{C}\left(H_{B \mid A}^{C}+H_{A \mid B}^{C}-H_{A B}^{\mid C}\right)-\left(v_{B} K_{\mid A}+v_{A} K_{\mid B}+K_{\mid A B}\right) \\
& +g_{A B}\left[r^{-3}\left(r^{3} K^{\mid C}\right)_{\mid C}-\frac{(l-1)(l+2)}{2 r^{2}} K-\frac{l(l+1)}{2 r^{2}} H_{C}^{C}+\left(H_{C \mid D}^{C}-2 H_{D \mid C}^{C}\right) v^{D}-\left(3 v_{C} v_{D}+2 v_{C \mid D}\right) H^{C D}\right], \\
& E_{A}\left[{ }^{\{n\}} h_{l}^{m}\right] \equiv \frac{1}{2}\left(H^{B}{ }_{B} v_{A}-H_{B \mid A}^{B}+H_{A}{ }^{B}{ }_{B}-K_{\mid A}\right), \\
& \tilde{E}\left[{ }^{\{n\}} h_{l}^{m}\right] \equiv \frac{1}{2}\left\{\left(H_{A B}-K g_{A B}\right)^{(4)} R^{A B}-\frac{l(l+1)}{2 r^{2}} H_{A}^{A}+H_{A \mid B}^{A}{ }^{B}-2 H_{B \mid A}^{A} v^{B}+H_{A \mid B}^{A} v^{B}-H_{\mid A B}^{A B}+K_{A}^{\mid A}+2 K_{\mid A} v^{A}\right\}, \\
& E\left[{ }^{[n\}} h_{l}^{m}\right] \equiv-\frac{1}{2} H_{A}^{A}, \\
& O_{A}\left[{ }^{[n\}} h_{l}^{m}\right] \equiv \frac{(l-1)(l+2)}{2 r^{2}} h_{A}-\frac{1}{2 r^{2}}\left[r^{4}\left(\frac{h_{A}}{r^{2}}\right)_{\mid C}-r^{4}\left(\frac{h_{C}}{r^{2}}\right)_{\mid A}\right]^{\mid C} \\
& O\left[{ }^{\{n\}} h_{l}^{m}\right] \equiv h^{A}{ }_{\mid A} .
\end{aligned}
$$

On the other hand, the linear parts of the equations for the perturbations of the energy-momentum conservation law, also in RW gauge, are the following:

$$
\begin{aligned}
& \left.L_{A}{ }^{[n\}} \psi_{l}^{m},\{n\} h_{l}^{m}\right] \equiv-\frac{l(l+1)}{r^{2}} \Psi_{A}-2 v_{A} \tilde{\Psi}+\frac{1}{r^{2}}\left(r^{2} \Psi_{A B}\right)^{\mid B}-\frac{1}{2} t^{B C} H_{B C \mid A}-\frac{r^{2}}{2} Q\left(r^{-2} K\right)_{\mid A}-\frac{1}{2} t_{A B} H_{C}^{C} C^{\mid B}-t_{A B} K^{\mid B} \\
& +\frac{1}{r^{2}}\left(r^{2} t_{A B} H^{B C}\right)_{\mid C} \\
& L\left[{ }^{\{n\}} \psi_{l}^{m},{ }^{\{n\}} h_{l}^{m}\right] \equiv \tilde{\Psi}-\frac{(l-1)(l+2)}{2 r^{2}} \Psi+\frac{1}{r^{2}}\left(r^{2} \Psi^{A}\right)_{\mid A}-\left(K-\frac{1}{2} H_{A}^{A}\right) \frac{Q}{2}-\frac{1}{2} H^{A B} t_{A B}, \\
& \tilde{L}\left[{ }^{\{n\}} \psi_{l}^{m},{ }^{\{n\}} h_{l}^{m}\right] \equiv \frac{1}{r^{2}}\left(r^{2} \psi^{A}\right)_{\mid A}-\frac{(l-1)(l+2)}{2 r^{2}} \psi-\frac{1}{2 r^{2}}\left(Q r^{2} h^{A}\right)_{\mid A}
\end{aligned}
$$




\section{APPENDIX E: COMPUTER IMPLEMENTATION}

Three MATHEMATICA packages have been constructed during the course of this investigation, respectively, called XPERT, HARMONICS, and XPERTGS. This appendix describes what is included in them. The three packages are based on the package XTENSOR [45] for abstract tensor computations, written by one of us, and also freely available.

The package XPERT implements the equations of Sec. II. In particular, there is a command Perturbation which plays the role of $\Delta$ and is able to give $n$ th-order perturbations from those at order $n-1$. The command Perturbation[expr, n] is recursively computed via Perturbation[Perturbation[expr, $n-1], 1]$. There is then the command GeneralPerturbation[expr, $n]$, which implements the expansion formulas for the metric (1), the inverse metric (8), the Christoffel symbols (12), the Riemann tensor (15), and the Ricci tensor and scalar. The general expansions are much faster than the recursive procedures, and, for instance, it is possible to produce within seconds the expressions of fifth-order perturbation theory with a small PC.

The package HARMONICS implements all the structures that have been defined on $S^{2}$ in Secs. III and IV, and Appendixes A, B, and $\mathrm{C}$ of this paper. The commands Pureorbital $[j, l, m][a, b, \ldots]$ and PureSpin $[j, \pm 1, m][a, b, \ldots]$ give, respectively, any pure-orbital or pure-spin harmonics, both in abstract form (in terms of the bases $t$ or $m$ ) or providing their components in any coordinate or noncoordinate basis. The generalized GS harmonics $Z$ and $X$ have also been defined, incorporating all their symmetries and properties (31)-(37), as well as the product formula (59).

The package XPERTGS needs the previous two packages and has three sections, closely following the mathematical structure of this article:

(1) Spherical background. The reduced manifold $M^{2}$ is defined with its metric $g[-\mathrm{A},-\mathrm{B}]$ (and corresponding derivative $C D[-A]$ ). The scalar field $r[]$ is also defined. The manifold $M^{4}$ is constructed as a product of $M^{2}$ and $S^{2}$; all tensors on $M^{4}$ can be blockdecomposed in their respective parts on those submanifolds.

(2) GS first order. First-order perturbations are defined and their equations computed using the gauge invariants (the total execution time is of the order of 2 minutes in a PC).

(3) GS second order. Second-order perturbations are defined and their equations computed and manipulated in order to simplify them as much as possible (the total execution time is of the order of 2 hours).

The packages XPERT and HARMONICS can be freely downloaded, under the GNU General Public License, from http://metric.iem.csic.es/Martin-Garcia/xAct/xPert.
[1] E. Seidel, Classical Quantum Gravity 21, S339 (2004).

[2] S. Chandrasekhar, The Mathematical Theory of Black Holes (Clarendon, Oxford, 1983).

[3] V. Mukhanov, H. Feldman, and R. Brandenberger, Phys. Rep. 215, 203 (1992).

[4] C. Gundlach, Phys. Rep. 376, 339 (2003).

[5] A. M. Khokhlov and I. D. Novikov, Classical Quantum Gravity 19, 827 (2002).

[6] C. Gundlach, G. Calabrese, I. Hinder, and J. M. MartínGarcía, Classical Quantum Gravity 22, 3767 (2005).

[7] N. Andersson, Classical Quantum Gravity 20, R105 (2003).

[8] T. Harada, H. Iguchi, and M. Shibata, Phys. Rev. D 68, 024002 (2003).

[9] S. Drasco and S. A. Hughes, Phys. Rev. D 73, 024027 (2006).

[10] R. J. Gleiser, C. O. Nicasio, R. H. Price, and J. Pullin, Phys. Rev. Lett. 77, 4483 (1996).

[11] M. Bruni, F. C. Mena, and R. Tavakol, Classical Quantum Gravity 19, L23 (2002).

[12] M. Campanelli and C. O. Lousto, Phys. Rev. D 59, 124022 (1999).

[13] A. Garat and R. H. Price, Phys. Rev. D 61, 044006 (2000).

[14] R. Gleiser, O. Nicasio, R. H. Price, and J. Pullin, Classical Quantum Gravity 13, L117 (1996).
[15] S. Chandrasekhar and V. Ferrari, Proc. R. Soc. A 433, 423 (1991).

[16] Y. Kojima, Phys. Rev. D 46, 4289 (1992).

[17] D. Garfinkle, C. Gundlach, and J. M. Martín-García, Phys. Rev. D 59, 104012 (1999).

[18] C. T. Cunningham, R.H. Price, and V. Moncrief, Astrophys. J. 236, 674 (1980).

[19] M. Mars, Classical Quantum Gravity 22, 3325 (2005).

[20] A. Passamonti, M. Bruni, L. Gualtieri, A. Nagar, and C. F. Sopuerta, Phys. Rev. D 73, 084010 (2006).

[21] U. H. Gerlach and U. K. Sengupta, Phys. Rev. D 19, 2268 (1979).

[22] U. H. Gerlach and U. K. Sengupta, Phys. Rev. D 22, 1300 (1980).

[23] C. Gundlach, Phys. Rev. D 57, R7075 (1998).

[24] K. Martel and E. Poisson, Phys. Rev. D 71, 104003 (2005).

[25] A. Nagar and L. Rezzolla, Classical Quantum Gravity 22, R167 (2005).

[26] C. Gundlach and J. M. Martín-García, Phys. Rev. D 61, 084024 (2000).

[27] J. M. Martín-García and C. Gundlach, Phys. Rev. D 64, 024012 (2001).

[28] T. Regge and J. Wheeler, Phys. Rev. 108, 1063 (1957).

[29] F. J. Zerilli, Phys. Rev. Lett. 24, 737 (1970). 
[30] V. Moncrief, Phys. Rev. D 9, 2707 (1974); 10, 1057 (1974).

[31] V. Moncrief, Ann. Phys. (N.Y.) 88, 323 (1974).

[32] O. Sarbach and M. Tiglio, Phys. Rev. D 64, 084016 (2001).

[33] F. J. Zerilli, J. Math. Phys. (N.Y.) 11, 2203 (1970).

[34] S. Wolfram, The Mathematica Book (Wolfram Media, 2003), 5th ed.

[35] More generally, one might consider metrics $\tilde{g}_{\mu \nu}(\epsilon)$ defined on manifolds $\mathcal{M}(\epsilon)$ that are related via an $\epsilon$ family of isomorphisms.

[36] One may also understand these expansions as a hierarchy of fields. At order $n$ in the hierarchy, two fields are identified if they differ by terms multiplied by $\epsilon^{n+1}$. In a more algebraic language, the domain of the fundamental quantities is the quotient ring $\mathbb{R}(\epsilon) / \epsilon^{n+1}$ [where $\mathbb{R}(\epsilon)$ is the ring of polynomials in the real variable $\epsilon$ with real coefficients].

[37] We also assume that the spacetime dependence of the metric and matter fields is (sufficiently) smooth.
[38] E. Newman and R. Penrose, J. Math. Phys. (N.Y.) 7, 863 (1966).

[39] A. R. Edmonds, Angular Momentum in Quantum Mechanics (Princeton University Press, Princeton, 1960), 2nd ed.

[40] A. Galindo and P. Pascual, Quantum Mechanics I, edited by W. Beiglbock (Springer-Verlag, Berlin, 1990).

[41] K. S. Thorne, Rev. Mod. Phys. 52, 299 (1980).

[42] J. N. Goldberg, A. J. Macfarlane, E. T. Newman, F. Rohrlich, and E. C. G. Sudarshan, J. Math. Phys. (N.Y.) 8, 2155 (1967).

[43] S. Sonego and M. Bruni, Commun. Math. Phys. 193, 209 (1998).

[44] V. Moncrief, J. Math. Phys. (N.Y.) 16, 493 (1975); 17, 1893 (1976).

[45] XTENSOR, a free package for tensor manipulations in MATHEMATiCA, José M. Martín-García. It can be downloaded under the GNU General Public License from http:// metric.iem.csic.es/Martin-Garcia/xAct. 